\title{
Polynomial Growth or Decay of Eigenfunctions of Second-order Elliptic Operators
}

By

\author{
Jun UCHIYAMA*
}

\section{§ 0 . Introduction}

In this paper we shall study the asymptotic behaviors as $|x| \rightarrow \infty$ of the not identically vanishing solution $u(x) \in H_{l o c}^{2}(\Omega)$ of secondorder elliptic equation in an unbounded domain $\Omega=\left\{x|| x \mid>R_{0}\right\}$ $\subset \mathbb{R}^{n}$

$$
\begin{aligned}
& -\sum_{i, j=1}^{n}\left(\frac{\partial}{\partial x_{i}}+\sqrt{-1} b_{i}(x)\right) a_{i j}(x)\left(\frac{\partial}{\partial x_{j}}+\sqrt{-1} b_{j}(x)\right) u(x) \\
& +\left(q_{1}(x)+q_{2}(x)\right) u(x)=0,
\end{aligned}
$$

where the matrix $\left(a_{i j}(x)\right)$ is uniformly positive definite, $b_{i}(x) \quad(1 \leq i$ $\leq n)$ and $q_{1}(x)$ are real-valued functions, and $q_{2}(x)$ is a complexvalued function. In case $a_{i j}(x)=\delta_{i j}$ (Kronecker's delta) we state the main parts of the assumptions: there exist some constants $\alpha, \beta, \gamma, \eta$ satisfying $\beta, \eta>0$ and $2-2 \beta<\gamma<\min \{2,2 \alpha\}$ such that

$$
\begin{aligned}
& \limsup _{r \rightarrow \infty} r^{2-2 \beta}\left[r \partial_{r} q_{1}(x)+\gamma q_{1}(x)\right. \\
& \left.\quad+(1+\eta)\left\{\frac{1}{2 \alpha-\gamma}\left|r q_{2}(x)\right|^{2}+\frac{1}{2-\gamma}|B(x) x|^{2}\right\}\right]<0,
\end{aligned}
$$

where $B(x)=\left(\partial_{i} b_{j}(x)-\partial_{j} b_{i}(x)\right)$ is an $n \times n$ matrix. Moreover there exist some constants $0<a<1,-\infty<\delta_{1}<\infty, C>0, \delta_{2} \leq \beta-2$ such that

$$
\begin{array}{lc}
\int_{\Omega}\left(q_{1}\right)_{-}|w|^{2} d x \leq a \int_{\Omega}|\nabla w|^{2} d x+C \int_{\Omega} r^{\delta_{1}}|w|^{2} d x & \text { for any } w(x) \in C_{0}^{\infty}(\Omega), \\
\left(\operatorname{Re}\left[q_{2}\right]\right)_{-}(x) \leq C r^{\delta_{2}} & \text { for }|x|>R_{0},
\end{array}
$$

where $(f)_{-}(x)=\max \{0,-f(x)\}$ for a real-valued function $f(x)$. More

Communicated by S. Matsuura, January 21, 1987.

* Kyoto Institute of Technology, Matsugasaki, Sakyoku, Kyoto 606, Japan. 
detailed conditions are stated in $\S 1$. Then by Theorem 1.3 in $\S 1$ we have for any $\varepsilon>0$

$$
\liminf _{R \rightarrow \infty} R^{\alpha+\max \left(0, \delta_{1}, \delta_{2}\right\}} \int_{R<|x|<R+\varepsilon}|u(x)|^{2} d x>0 .
$$

Moreover if $\alpha+\max \left\{0, \delta_{1}, \delta_{2}\right\} \leq 1$, then $u(x) \notin L^{2}(\Omega)$.

This result can be recognized as a generalization of Kato [5], Agmon [1, Theorem 3], Agmon [2, Theorems 3, 4 and 5], IkebeUchiyama [4], Uchiyama [7], Mochizuki [6], Eastham-Kalf [3, Theorems 6.3.3 and 6.4.2] and Yamada [12] from following points of view. [1] and [12] did not give their own complete proof. The term $a_{i j}(x)$ is lacking in [5], [1], [2], [7], [3] and [12]. The term $b_{i}(x)$ is lacking in [5], [1], [2] and [7]. In [5], [2], [4] and [12] it was assumed that $q_{1}(x)<0$ for $r>R_{0}$. So they are not applicable to atomic type many body potential treated in Example 5.3 and Remark 5.4 in §5. In [5], [4], [7] and [6] $\delta_{1}$ was restricted to satisfy $\delta_{1} \leq 0$. Except for [1] and [3, Theorem 6.3.3], it was difficult to treat Examples 5.1 5.8 in one theorem. But in [3] only the case $\beta=1$ was treated. So in order to show $u(x) \notin L^{2}(\Omega)$, they assumed $\delta_{1}<1$ and $\delta_{2} \leq-1$, which was needed to satisfy $\alpha+\max \left\{0, \delta_{1}, \delta_{2}\right\} \leq 1$ (noting $2-2 \beta=0<\gamma<2 \alpha$ ). And in case $\delta_{1} \geq 1$ they needed another theorem [3, Theorem 6.4.2], which corresponds to Example 5.1 in $\S 5$ and [2, Theorem 5].

Here we note that [1], [2], [7] and [12] treated the critical case $2-2 \beta \leq \gamma \leq 2 \alpha$ and they gave better estimates than ours under more strict conditions on $q_{2}(x)$ and curl $b(x)$.

The introduction of $\beta>0$ in the above assumptions can be found in [1]. Other articles treated only the case $\beta=1$ (i. e. $2-2 \beta=0$ ), and are not applicable to Example 5.2 in $\S 5$. Such conditions depending on $\beta$ are also found in Uchiyama $[8,9,10]$, which deal the exponential decay of $u(x)$ at infinity.

In $\S 1$ the Assumptions and main results are explained. $\S 2$ is the preliminary stage of the proofs of the theorems. The identity given in Lemma 2.8 is fully used in $\S 3$ and $\S 4$. In $\S 5$ we give several applications of Theorems 1.3 and 1.4. Some of Examples in $\S 5$ were also considered in $[8,9,10]$ which showed

$$
\lim _{R \rightarrow \infty} \exp \left\{R^{\varepsilon}\right\} \int_{R<|x|<R+1}|u(x)|^{2} d x=+\infty \quad \text { for any } \varepsilon>0 .
$$


But here for such solutions $u(x)$ we shall show that

$$
\lim _{R \rightarrow \infty} \inf ^{\mu} \int_{R<|x|<R+1}|u(x)|^{2} d x>0
$$

holds, and we shall calculate $\mu$.

\section{§1. Assumptions and Main $\mathbb{R e s u l t s}$}

At first we shall list the notations which will be freely used in the sequel, and are the same as given in Uchiyama [8].

\section{Notations:}

$\langle\xi, \eta\rangle=\xi_{1} \eta_{1}+\cdots+\xi_{n} \eta_{n} \quad$ for $\xi=\left(\xi_{1}, \ldots, \xi_{n}\right), \eta=\left(\eta_{1}, \ldots, \eta_{n}\right) \in \mathbb{C}^{n}$;

$|\xi|=(\langle\xi, \bar{\xi}\rangle)^{1 / 2} \quad$ for $\xi \in \mathbb{C}^{n}$;

$\hat{x}=x /|x|$ and $r=|x| \quad$ for $x=\left(x_{1}, \ldots, x_{n}\right) \in R^{n}$;

$\partial_{j}=\partial / \partial x_{j}$ and $\partial_{r}=\partial / \partial r$

$D_{j}=\partial_{j}+\sqrt{-1} b_{j}(x)$ and $D=\left(D_{1}, \ldots, D_{n}\right)$;

$\nabla f=\left(\partial_{1} f, \ldots, \partial_{n} f\right)$ for a scalar valued function $f(x)$;

$\operatorname{div} g=\partial_{1} g_{1}+\cdots+\partial_{n} g_{n}$ for a vector valued function $g(x)=$ $\left(g_{1}(x), \ldots, g_{n}(x)\right)$;

$A=A(x)=\left(a_{i j}(x)\right)$ is an $n \times n$ matrix;

$B=B(x)=\operatorname{curl} b(x)=\left(\partial_{i} b_{j}(x)-\partial_{j} b_{i}(x)\right)$ is an $n \times n$ matrix;

$(f)_{ \pm}(x)=\max \{0, \pm f(x)\} \geq 0 \quad$ for a real-valued function $f(x)$;

$\operatorname{supp}[f]$ denotes the closure of $\{x \mid f(x) \neq 0\}$;

$C^{j}(\Omega)$ denotes the class of $j$-times continuously differentiable functions ;

$C_{0}^{\infty}(\Omega)=\left\{f(x) \mid\right.$ for any $j=0,1,2, \ldots, f \in C^{j}(\Omega)$ and $\operatorname{supp}[f]$ is a compact set in $\Omega\}$;

$L^{p}(\Omega)=\left\{\left.f(x)\left|\int_{\Omega}\right| f(x)\right|^{p} d x<\infty\right\} \quad$ for $p \geq 1$;

$L_{\mathrm{loc}}^{p}(\Omega)=\left\{f(x) \mid\right.$ for any compact set $\left.K \subset \Omega, \int_{k}|f(x)|{ }^{p} d x<\infty\right\}$ for $p \geq 1$;

$H^{m}(\Omega)$ denotes the class of $L^{2}$-functions in $\Omega$ such that all distribution derivatives up to $m$ belong to $L^{2}(\Omega)$;

$H_{\mathrm{loc}}^{m}(\Omega)$ denotes the class of $L_{\mathrm{loc}}^{2}-$ functions in $\Omega$ such that all distribution derivatives up to $m$ belong to $L_{\text {loc }}^{2}(\Omega)$;

$\left(\int_{|x|=t}-\int_{|x|=s}\right) f(x) d S=\int_{|x|=t} f(x) d S-\int_{|x|=s} f(x) d S$ 。 
Next we shall state the conditions required in the theorems.

\section{Assumptions :}

(A1) each $a_{i j}(x) \in C^{2}(\Omega)$ is a real-valued function;

(A2) $a_{i j}(x)=a_{j i}(x)$;

(A3) $a_{i j}(x) \rightarrow \delta_{i j}$ as $|x| \rightarrow \infty$;

(A4) there exists some constant $C_{1} \geq 1$ such that for any $x \in \Omega$ and any $\xi \in \mathbb{C}^{n}$ we have

$$
C_{1}^{-1}|\xi|^{2} \leq\langle A(x) \xi, \bar{\xi}\rangle \leq C_{1}|\xi|^{2} ;
$$

(A5) $\partial_{l} a_{i j}(x)=o\left(r^{-1}\right)$ as $|x| \rightarrow \infty$;

(B1) each $b_{i}(x)$ is a real-valued function;

(B2) for any $w \in H_{\mathrm{loc}}^{1}(\Omega)$ we have $\left|b_{i}\right|^{2}|w|^{2},\left|\partial_{i} b_{j}\right|^{2}|w|^{2} \in L_{\mathrm{loc}}^{1}(\Omega)$;

(C1) $q_{1}(x)$ is a real-valued function;

(C2) for any $w \in H_{\mathrm{loc}}^{1}(\Omega)$ we have $\left|q_{1}\right||w|^{2} \in L_{\mathrm{loc}}^{1}(\Omega)$;

(C3) for any $w \in H_{\mathrm{loc}}^{1}(\Omega)$ we have $\left|\nabla q_{1}\right||w|^{2} \in L_{\mathrm{loc}}^{1}(\Omega)$;

(D1) $q_{2}(x)$ is a complex-valued function;

(D2) for any $w \in H_{\mathrm{loc}}^{1}(\Omega)$ we have $\left|q_{2}\right||w|^{2} \in L_{\mathrm{loc}}^{1}(\Omega)$;

(E) $\quad \Omega$ is a domain in $\mathbb{R}^{n}$ and there exists some constant $R_{0}>1$ such that $\Omega \supset\left\{x|| x \mid>R_{0}\right\}$;

(F) there exist some constants $\alpha, \beta, \delta, \eta$ satisfying $0<\delta<\beta, \eta>0$ and some real-valued function $\gamma(x) \in C^{1}(\Omega)$ such that (F1) $\sim(\mathrm{F} 5)$ hold.

(F1) $\alpha \geq \frac{1}{2} \gamma(x)+\delta$ for $|x|>R_{0}$;

(F2) $2-2 \beta+\delta \leq \gamma(x) \leq 2-\delta \quad$ for $|x|>R_{0}$;

$$
\begin{aligned}
& \limsup _{r \rightarrow \infty} r^{2-2 \beta}\left[r\left\langle A(x) \nabla q_{1}(x), \hat{x}\right\rangle+\gamma(x)\langle A(x) \hat{x}, \hat{x}\rangle q_{1}(x)\right. \\
& +(1+\eta)\left\{\frac{1}{2 \alpha-\gamma(x)}\left|r q_{2}(x)\right|^{2}\right. \\
& \left.\left.+\frac{1}{2-\gamma(x)}\langle A(x) \hat{x}, \hat{x}\rangle^{-1}\langle A(x) B(x) A(x) x, B(x) A(x) x\rangle\right\}\right]<0
\end{aligned}
$$

(F4) $\partial_{l} \partial_{m} a_{i j}(x)=o\left(r^{\beta-2}\right) \quad$ as $|x| \rightarrow \infty$;

(F5) $\nabla \gamma(x)=o\left(r^{\beta-1}\right) \quad$ as $|x| \rightarrow \infty$;

(G1) there exist some constants $0<a<1,-\infty<\delta_{1}<\infty$ and $C_{2}>0$ such that for any $w \in C_{0}^{\infty}(\Omega)$ we have 


$$
\int_{\Omega}\left(q_{1}\right)-|w|^{2} d x \leq a \int_{\Omega}|\nabla w|^{2} d x+C_{2} \int_{\Omega} r^{\delta_{1}}|w|^{2} d x
$$

(G2) there exist some constants $\delta_{2} \leq \beta-2$ and $C_{3}>0$ such that for any $|x|>R_{0}$ we have

$$
\left(\operatorname{Re}\left[q_{2}\right]\right)_{-}(x) \leq C_{3} r^{\delta_{2}},
$$

where $\operatorname{Re}[z]$ means the real part of $z \in \mathbb{C}$.

Now we have the

Theorem 1. 1. Let $u(x)$ satisfy

$$
\left(^{*}\right):\left\{\begin{array}{l}
-\langle D, A D u\rangle+\left(q_{1}(x)+q_{2}(x)\right) u(x)=0 \text { in } \Omega, \\
u(x) \in H_{\text {loc }}^{2}(\Omega), \\
\operatorname{supp}[u] \text { is not a compact set in } \bar{\Omega} \text { (closure of } \Omega \text { ). }
\end{array}\right.
$$

Let conditions $(\mathrm{A}) \sim(\mathrm{G})$ hold. Then we have

$$
\left.\lim _{R \rightarrow \infty} \inf ^{\alpha} \int_{|x|=R}\left[|\langle A D u, \hat{x}\rangle|^{2}+\left(r^{-2}+q_{1}\right)_{-}\right)|u|^{2}\right] d S>0,
$$

and for any $\varepsilon>0$

$$
\lim _{R \rightarrow \infty} \inf ^{\alpha+\max \left(0, \delta_{1}, \delta_{2}\right\}} \int_{R<|x|<R+\varepsilon}|u|^{2} d x>0 .
$$

Corollary 1.2. Let $u(x)$ satisfy $\left({ }^{*}\right)$, and let conditions $(\mathrm{A}) \sim(\mathrm{G})$ hold. If

$$
\alpha+\max \left\{0, \delta_{1}, \delta_{2}\right\} \leq 1,
$$

then we have $u(x) \notin L^{2}(\Omega)$.

Now we shall consider the more special case under the weaker conditions.

Theorem 1.3. Let $u(x)$ satisfy

$$
(* *):\left\{\begin{array}{l}
-\langle D, D u\rangle+\left\{q_{1}(x)+q_{2}(x)\right\} u(x)=0 \text { in } \Omega, \\
u \in H_{\mathrm{loc}}^{2}(\Omega), \\
\operatorname{supp}[u] \text { is not a compact set in } \bar{\Omega} .
\end{array}\right.
$$

We assume $(\mathrm{B}) \sim(\mathrm{G})$ with $a_{i j}(x)=\delta_{i j}$ except for (C3). Instead of (C3) we assume 
(C3)' for any $w \in H_{\mathrm{loc}}^{1}(\Omega)$ we have $\left|\partial_{r} q_{1}\right||w|^{2} \in L_{\mathrm{loc}}^{1}(\Omega)$.

Then we have

$$
\lim _{R \rightarrow \infty} \inf ^{\alpha} \int_{|x|=R}\left[|\langle D u, \hat{x}\rangle|^{2}+\left\{r^{-2}+\left(q_{1}\right)_{-}\right\}|u|^{2}\right] d S>0,
$$

and for any $\varepsilon>0$

$$
\lim _{R \rightarrow \infty} \inf ^{\alpha+\max \left(0, \delta_{1}, \delta_{2}\right)} \int_{R<|x|<R+\varepsilon}|u|^{2} d x>0 .
$$

Moreover if

$$
\alpha+\max \left\{0, \delta_{1}, \delta_{2}\right\} \leq 1,
$$

then we have $u(x) \notin L^{2}(\Omega)$.

Lastly we shall consider the most special case under the weakest conditions.

Theorem 1.4. Let $u(x)$ satisfy

$$
(* * *):\left\{\begin{array}{l}
-\Delta u(x)+\left\{q_{1}(x)+q_{2}(x)\right\} u(x)=0 \text { in } \Omega, \\
u \in H_{\mathrm{loc}}^{2}(\Omega), \\
\operatorname{supp}[u] \text { is not a compact set in } \bar{\Omega},
\end{array}\right.
$$

where $\Delta$ is a Laplacian in $\mathbb{R}^{n}$. We assume $(\mathrm{C}) \sim(\mathrm{G})$ with $a_{i j}(x)=\delta_{i j}$ and $b_{i}(x)=0$ except for (C3), (F2) and (F5). Instead of (C3), (F2) and $(\mathrm{F} 5)$ we assume $(\mathrm{C} 3)^{\prime}$, and

(F2)' $2-2 \beta+\delta \leq \gamma(x) \leq 2$;

(F5)' $\partial_{r} \gamma(x)=o\left(r^{\beta-1}\right)$ as $|x| \rightarrow \infty$ and

$$
\begin{aligned}
& \left|\left(\nabla-\hat{x} \partial_{r}\right) \gamma(x)\right| \leq\{2-r(x)\}^{1 / 2} p(x), \text { where } p(x)=o\left(r^{\beta-1}\right) \text { as } \\
& |x| \rightarrow \infty .
\end{aligned}
$$

Then we have

$$
\lim _{R \rightarrow \infty} \inf ^{\alpha} \int_{|x|=R}\left[\left|\partial_{r} u\right|^{2}+\left\{r^{-2}+\left(q_{1}\right)_{-}\right\}|u|^{2}\right] d S>0,
$$

and for any $\varepsilon>0$

$$
\lim _{R \rightarrow \infty} \inf R^{\left.\alpha+\max i 0, \delta_{1}, \delta_{2}\right\}} \int_{R<|x|<R+\varepsilon}|u|^{2} d x>0 .
$$

Moreover if

$$
\alpha+\max \left\{0, \delta_{1}, \delta_{2}\right\} \leq 1,
$$

then we have $u(x) \notin L^{2}(\Omega)$. 
Remark 1.5. (F2)' and (F5)' are weaker conditions than (F2) and (F5). In fact $\gamma(x)=2$ (constant) satisfies (F2)' and (F5)', and does not satisfy (F2) and (F5).

\section{§2. Preliminary Lemmas}

In this section all the conditions $(A) \sim(G)$ are assumed. And let $u(x)$ satisfy $(*)$.

\section{Lemma 2.1. We have}

(1) $\langle A(x) \hat{x}, \hat{x}\rangle=1+o(1)$ as $|x| \rightarrow \infty$,

(2) $\partial_{i}(\langle A(x) \hat{x}, \hat{x}\rangle)=o\left(r^{-1}\right)$ as $|x| \rightarrow \infty$, and

$\partial_{i}\left(\langle A(x) \hat{x}, \hat{x}\rangle^{-1}\right)=o\left(r^{-1}\right)$ as $|x| \rightarrow \infty$,

(3) $\partial_{i} \partial_{j}(\langle A(x) \hat{x}, \hat{x}\rangle)=o\left(r^{\beta-2}\right)$ as $|x| \rightarrow \infty$, and

$\partial_{i} \partial_{j}\left(\langle A(x) \hat{x}, \hat{x}\rangle^{-1}\right)=o\left(r^{\beta-2}\right)$ as $|x| \rightarrow \infty$,

(4) $\operatorname{div}(A(x) \hat{x})=(n-1) r^{-1}+o\left(r^{-1}\right)$ as $|x| \rightarrow \infty$,

(5) $\partial_{i}(\operatorname{div}(A(x) \hat{x}))=o\left(r^{\beta-2}\right)$ as $|x| \rightarrow \infty$.

Proof. Noting (A3), (A5), (F4) and $\beta>0$, we have the assertions by direct calculations. In fact we have

$$
\langle A \hat{x}, \hat{x}\rangle=1+\langle(A-E) \hat{x}, \hat{x}\rangle,
$$

where $E=\left(\delta_{i j}\right)$ is the $n \times n$ identity matrix,

$$
\begin{aligned}
& \nabla(\langle A \hat{x}, \hat{x}\rangle)=\langle(\nabla A) \hat{x}, \hat{x}\rangle+2 r^{-1}\{(A-E) \hat{x}-\hat{x}\langle(A-E) \hat{x}, \hat{x}\rangle\}, \\
& \partial_{i} \hat{\partial}_{j}(\langle A \hat{x}, \hat{x}\rangle)=\sum_{k, l=1}^{n}\left\{\left(\partial_{i} \partial_{j} a_{k l}(x)\right) \hat{x}_{k} \hat{x}_{l}+\left(\partial_{i} a_{k l}(x)\right) \partial_{j}\left(\hat{x}_{k} \hat{x}_{l}\right)\right. \\
& \left.+\left(\partial_{j} a_{k l}(x)\right) \partial_{i}\left(\hat{x}_{k} \hat{x}_{l}\right)+a_{k l}(x) \partial_{i} \partial_{j}\left(\hat{x}_{k} \hat{x}_{l}\right)\right\}, \\
& \partial_{i} \hat{x}_{k}=O\left(r^{-1}\right) \text { and } \partial_{i} \partial_{j} \hat{x}_{k}=O\left(r^{-2}\right) \text { as }|x| \rightarrow \infty \text {, } \\
& \operatorname{div}(A \hat{x})=(n-1) r^{-1}+\operatorname{div}\{(A-E) \hat{x}\} \text {, } \\
& \partial_{i}\{\operatorname{div}(A \hat{x})\}=\sum_{k, l=1}^{n}\left[\left\{\partial_{i} \partial_{k} a_{k l}(x)\right\} \hat{x}_{l}+\left\{\partial_{k} a_{k l}(x)\right\} \partial_{i} \hat{x}_{l}\right. \\
& \left.+\left\{\partial_{i} a_{k l}(x)\right\} \partial_{k} \hat{x}_{l}+a_{k l}(x) \partial_{i} \partial_{k} \hat{x}_{l}\right] \text {. }
\end{aligned}
$$

Lemma 2.2. For any $x \in \Omega$ and any $\xi \in \mathbb{C}^{n}$ we have

$$
\begin{gathered}
\langle A(x) \xi, \bar{\xi}\rangle-\langle A(x) \hat{x}, \hat{x}\rangle^{-1}|\langle A(x) \xi, \hat{x}\rangle|^{2} \\
=\left\langle A(x)\left\{\xi-\hat{x}\langle A(x) \hat{x}, \hat{x}\rangle^{-1}\langle A(x) \xi, \hat{x}\rangle\right\},\right. \\
\left.\left\{\bar{\xi}-\hat{x}\langle A(x) \hat{x}, \hat{x}\rangle^{-1}\langle A(x) \bar{\xi}, \hat{x}\rangle\right\}\right\rangle \geq 0 .
\end{gathered}
$$


Proof. By direct calculation we have the assertion.

Lemma 2.3. There exist some constants $R_{1}>R_{0}$ and $C_{4}>0$ such that for any real-valued function $\phi(r) \in C_{0}^{1}\left(R_{1}, \infty\right)$ we have

$$
\int_{\Omega} \phi(r)^{2}\left\{|D u|^{2}+\left(q_{1}\right)-|u|^{2}\right\} d x \leq C_{4} \int_{\Omega}\left\{r^{\max \left(\delta_{1}, \delta_{2}\right\}} \phi(r)^{2}+\phi^{\prime}(r)^{2}\right\}|u|^{2} d x .
$$

Proof. By (A3), (A4) and (G1), there exist some $R_{1}>R_{0}$ and $\varepsilon>0$ such that for any $r \geq R_{1}$ and any $\xi \in \boldsymbol{C}^{n}$ we have

$$
\begin{aligned}
& (1-\varepsilon)|\xi|^{2} \leq\langle A(x) \xi, \bar{\xi}\rangle \leq(1+\varepsilon)|\xi|^{2}, \\
& (1-\varepsilon)^{2}-(1+\varepsilon) a>0 .
\end{aligned}
$$

Since $u(x)$ satisfies $(*)$, we have by integration by parts for $\phi(r) \in$ $C_{0}^{1}\left(R_{1}, \infty\right)$

$$
\begin{aligned}
0= & \operatorname{Re} \int_{\Omega}\left[-\langle D, A D u\rangle+\left(q_{1}+q_{2}\right) u\right] \phi(r)^{2} \overline{u(x)} d x \\
= & \int_{\Omega}\left[\phi^{2}\langle A D u, \overline{D u}\rangle+2 \phi \phi^{\prime} \operatorname{Re}[\langle A D u, \hat{x}\rangle \bar{u}]\right. \\
& \left.+\phi^{2}\left(q_{1}+\operatorname{Re}\left[q_{2}\right]\right)|u|^{2}\right] d x \\
\geq & \int_{\Omega}\left[\phi^{2}\langle A D u, \overline{D u}\rangle-\varepsilon \phi^{2}\langle A \hat{x}, \hat{x}\rangle-1|\langle A D u, \hat{x}\rangle|^{2}\right. \\
& \left.-\varepsilon^{-1} \phi^{\prime 2}\langle A \hat{x}, \hat{x}\rangle|u|^{2}-\phi^{2}\left\{\left(q_{1}\right)_{-}+\left(\operatorname{Re}\left[q_{2}\right]\right)-\right\}|u|^{2}\right] d x \\
\geq & (1-\varepsilon)^{2} \int_{\Omega} \phi^{2}|D u|^{2} d x-\int_{\Omega}\left[\varepsilon^{-1}(1+\varepsilon) \phi^{\prime 2}|u|^{2}+\phi^{2}\left\{\left(q_{1}\right)_{-}+C_{3}{ }^{\delta^{2}}{ }^{2}\right\}|u|^{2}\right] d x,
\end{aligned}
$$

where we use Lemma 2.2. For any $\eta>0$ let $u_{\eta}(x)=\left\{|u(x)|^{2}+\eta^{2}\right\}^{1 / 2}$. Since $|u| \leq\left|u_{\eta}\right|$ and $u_{\eta} \nabla u_{\eta}=\operatorname{Re}[\bar{u} \nabla u]=\operatorname{Re}[\bar{u} D u]$, we have

$$
\begin{aligned}
& \left|\nabla u_{\eta}\right| \leq|D u|, \\
& \phi(r) u_{\eta}(x) \in H^{1}(\Omega), \\
& \operatorname{supp}\left[\phi(r) u_{\eta}(x)\right] \text { is a compact set in } \Omega .
\end{aligned}
$$

By (Gl) and limiting procedure we have for any $w \in H^{1}(\Omega)$ satisfying that $\operatorname{supp}[w]$ is a compact set in $\Omega$

$$
\int_{\Omega}\left(q_{1}\right)-|w|^{2} d x \leq a \int_{\Omega}|\nabla w|^{2} d x+C_{2} \int_{\Omega} r^{\delta_{1}}|w|^{2} d x .
$$

Then we have

$$
\begin{aligned}
\int_{\Omega}\left(q_{1}\right)_{-} \phi^{2}\left|u_{\eta}\right|^{2} d x \leq & a \int_{\Omega}\left|\nabla\left(\phi u_{\eta}\right)\right|^{2} d x+C_{2} \int_{\Omega} r^{\delta_{1}}\left|\phi u_{\eta}\right|^{2} d x \\
\leq & (1+\varepsilon) a \int_{\Omega} \phi^{2}|D u|^{2} d x+\int_{\Omega}\left\{\varepsilon^{-1}(1+\varepsilon) a \phi^{\prime 2}\right. \\
& \left.+C_{2} r^{\delta_{1}} \phi^{2}\right\}\left|u_{\eta}\right|^{2} d x .
\end{aligned}
$$


Let $\eta \downarrow 0$. Since $q_{1} \in L_{\mathrm{loc}}^{1}(\Omega)$ by (C2), we have by Lebesgue's dominated convergence theorem

$$
\begin{gathered}
\int_{\Omega}\left(q_{1}\right)_{-} \phi^{2}|u|^{2} d x \leq(1+\varepsilon) a \int_{\Omega} \phi^{2}|D u|^{2} d x \\
+\int_{\Omega}\left\{\varepsilon^{-1}(1+\varepsilon) a \phi^{\prime 2}+C_{2} r^{\delta_{1}} \phi^{2}\right\}|u|^{2} d x
\end{gathered}
$$

Then we have

$$
\int_{\Omega} \phi^{2}|D u|^{2} d x \leq C_{5} \int_{\Omega}\left\{r^{\max \left(\delta_{1}, \delta_{2}\right\}} \phi^{2}+\phi^{\prime 2}\right\}|u|^{2} d x
$$

where $C_{5}=\left\{(1-\varepsilon)^{2}-(1+\varepsilon) a\right\}^{-1} \max \left\{C_{2}+C_{3}, \varepsilon^{-1}(1+\varepsilon)(1+a)\right\}$. Therefore we have

$$
\int_{\Omega} \phi^{2}\left\{|D u|^{2}+\left(q_{1}\right)-|u|^{2}\right\} d x \leq C_{4} \int_{\Omega}\left\{r^{\max \left(\delta_{1}, \delta_{2}\right)} \phi^{2}+\phi^{\prime 2}\right\}|u|^{2} d x
$$

where $C_{4}=C_{5}+(1+\varepsilon) a C_{5}+\max \left\{C_{2}, \varepsilon^{-1}(1+\varepsilon) a\right\}>0$ 。

Lemma 2.4. There exists some constant $C_{6}>0$ such that for any realvalued function $\zeta(r) \in C^{1}\left[R_{1}, \infty\right)$ satisfying

$$
\begin{aligned}
& \int_{|x|>R_{1}} r^{\left.\max \mid \delta_{1}, \delta_{2}\right\}} \zeta(r)^{2}|u(x)|^{2} d x<\infty, \\
& \int_{|x|>R_{1}} \zeta^{\prime}(r)^{2}|u(x)|^{2} d x<\infty, \\
& \liminf _{t \rightarrow \infty} \int_{t<|x|<t+1} \zeta(r)^{2}|u(x)|^{2} d x=0,
\end{aligned}
$$

and for any $R \geq R_{1}$ (where $R_{1}$ is the one given in Lemma 2.3), we have

$$
\begin{aligned}
\int_{|x|>R+1} \zeta(r)^{2}\left\{|D u|^{2}+\left(q_{1}\right)-|u|^{2}\right\} d x \leq & C_{6} \int_{|x|>R}\left(r^{\max \left(\delta_{1}, \delta_{2} \mid\right.} \zeta^{2}+\zeta^{\prime 2}\right)|u|^{2} d x \\
& +C_{6} \int_{R<|x|<R+1} \zeta^{2}|u|^{2} d x .
\end{aligned}
$$

Proof. For any $R \geq R_{1}$ and $t>R+2$ we choose $\xi_{R t}(r) \in C_{0}^{\infty}(R, t)$ satisfying $\xi_{R t}(r)=1$ for $R+1 \leq r \leq t-1$ and $0 \leq \xi_{R t}(r) \leq 1$ for $R \leq r \leq t$. At the same time we can assume that there exists some constant $C_{7}>0$ such that for any $R \geq R_{1}, t>R+2$ and $r \geq R_{1}$ we have $\left|\xi_{R t}^{\prime}(r)\right| \leq C_{7}$ 。 Then by Lemma 2.3 we have for any $R \geq R_{1}$ and $t>R+2$, noting $\xi_{R t}^{\prime}(r)=0$ for $R+1<r<t-1$,

$$
\int_{R+1<|x|<t-1} \zeta(r)^{2}\left\{|D u|^{2}+\left(q_{1}\right)-|u|^{2}\right\} d x
$$




$$
\begin{aligned}
& \leq \int_{\Omega}\left(\xi_{R t} \zeta\right)^{2}\left\{|D u|^{2}+\left(q_{1}\right)-|u|^{2}\right\} d x \\
& \leq C_{4} \int_{\Omega}\left\{r^{\left.\max \mid \delta_{1}, \delta_{2}\right)}\left(\xi_{R t} \zeta\right)^{2}+\left(\xi_{R t}^{\prime} \zeta+\xi_{R t} \zeta^{\prime}\right)^{2}\right\}|u|^{2} d x \\
& \leq C_{4} \int_{R<|x|<t}\left(r^{\max \left(\hat{\delta}_{1}, \delta_{2} \mid\right.} \zeta^{2}+2 \zeta^{\prime 2}\right)|u|^{2} d x \\
& \quad+2 C_{4} C_{7}^{2}\left\{\int_{R<|x|<R+1} \zeta^{2}|u|^{2} d x+\int_{t-1<|x|<t} \zeta^{2}|u|^{2} d x\right\} .
\end{aligned}
$$

Letting $t \rightarrow \infty$ along the suitable subsequence and letting $C_{6}=\max$ $\left\{2 C_{4}, 2 C_{4} C_{7}^{2}\right\}>0$, we have the assertion.

Definition 2.5. For real-valued functions $\rho(r) \in C^{2}\left(R_{0}, \infty\right), f(x)$ $\in C^{1}(\Omega)$ and $g(x) \in C^{1}(\Omega)$, let

$$
\begin{aligned}
& v(x)=e^{\rho(r)} u(x), \\
& k_{1}(x)=-\left\{\rho^{\prime}(r)\right\}^{2}\langle A(x) \hat{x}, \hat{x}\rangle, \\
& k_{2}(x)=\rho^{\prime \prime}(r)\langle A(x) \hat{x}, \hat{x}\rangle+\rho^{\prime}(r) \operatorname{div}\{A(x) \hat{x}\}, \\
& F(t ; \rho, f, g)=\int_{|x|=t}\left[f ( x ) \langle A ( x ) \hat { x } , \hat { x } \rangle \left\{2\langle A(x) \hat{x}, \hat{x}\rangle^{-1}|\langle A D v, \hat{x}\rangle|^{2}\right.\right. \\
& \left.\left.\quad-\langle A D v, \overline{D v}\rangle-\left(q_{1}+k_{1}\right)|v|^{2}\right\}+g(x) \operatorname{Re}[\langle A D v, \hat{x}\rangle \bar{v}]\right] d S .
\end{aligned}
$$

The meaning of $F(t ; \rho, f, g)$ can be cleared by Lemma 2.8 .

Lemma 2.6. We have for $t>R_{0}$

$$
\begin{aligned}
F(t ; \rho, f, g)= & e^{2 \rho(t)} \int_{! x \mid=t}\left[f(x)\left\{2|\langle A D u, \hat{x}\rangle|^{2}-\langle A \hat{x}, \hat{x}\rangle\langle A D u, \overline{D u}\rangle\right\}\right. \\
& +\left\{2 \rho^{\prime} f\langle A \hat{x}, \hat{x}\rangle+g\right\} \operatorname{Re}[\langle A D u, \hat{x}\rangle \bar{u}] \\
& \left.+\left\{2 f \rho^{\prime}\langle A \hat{x}, \hat{x}\rangle^{2}+\left(g \rho^{\prime}-f q_{1}\right)\langle A \hat{x}, \hat{x}\rangle\right\}|u|^{2}\right] d S .
\end{aligned}
$$

Proof. Noting Definition 2.5 we have the assertion by direct calculation.

To prove Lemma 2.8 we use the following.

Lemma 2.7. Let $w, \partial_{i} w \in L_{\mathrm{loc}}^{1}(\Omega)$, where $\partial_{i} w$ is the derivative in the sense of distribution. Then for any $t>R_{0}, \int_{|x|=t} \hat{x}_{i} w d S$ exists (by choosing suitable representative for $w$, if necessary), and we have for any $t>s>R_{0}$ 


$$
\int_{s<|x|<t} \partial_{i} w d x=\int_{|x|=t} \hat{x}_{i} w d S-\int_{|x|=s} \hat{x}_{i} w d S .
$$

Proof. See Eastham and Kalf [3, p. 239].

Lemma 2.8. For any $t>s>R_{0}$ we have

$$
\begin{aligned}
F(t ; \rho, & f, g)-F(s ; \rho, f, g) \\
= & \int_{s<|x|\langle t}[2 \operatorname{Re}[\langle A D v, \nabla f\rangle\langle A \overline{D v}, \hat{x}\rangle]+\{g-\langle A \nabla f, \hat{x}\rangle \\
& -f \operatorname{div}(A \hat{x})\}\langle A \hat{x}, \hat{x}\rangle^{-1}|\langle A D v, \hat{x}\rangle|^{2}+\left\{2 r^{-1} f\langle\operatorname{A} \hat{x}, \hat{x}\rangle\right. \\
& +g-\langle A \nabla f, \hat{x}\rangle-f \operatorname{div}(A \hat{x})\}\{\langle A D v, \overline{D v}\rangle \\
& \left.-\langle A \hat{x}, \hat{x}\rangle^{-1}|\langle A D v, \hat{x}\rangle|^{2}\right\}+2 r^{-1} f\left\{|A D v|^{2}-\langle A \hat{x}, \hat{x}\rangle\langle A D v, \overline{D v}\rangle\right\} \\
& +2 f \operatorname{Re}[\langle(\langle A D v, \nabla\rangle A) \overline{D v}, \hat{x}\rangle]-f \operatorname{Re}[\langle(\langle\hat{x}, A \nabla\rangle A) D v, \overline{D v}\rangle] \\
& -2 f \operatorname{Re}\left[\sqrt{-1}\left\langle A B A \hat{x},\left(D v-\hat{x}\langle A \hat{x}, \hat{x}\rangle^{-1}\langle A D v, \hat{x}\rangle\right)\right\rangle \bar{v}\right] \\
& +2 f \operatorname{Re}\left[\langle A D v, \hat{x}\rangle \overline{q_{2} v}\right]+\operatorname{Re}[\langle A D v, \nabla g\rangle \bar{v}]+\{(g-\langle A \nabla f, \hat{x}\rangle \\
& \left.\left.-f \operatorname{div}(A \hat{x})) q_{1}-f\left\langle A \nabla q_{1}, \hat{x}\right\rangle+g \operatorname{Re}\left[q_{2}\right]\right\}|v|^{2}\right] d x \\
& +\int_{s<! x \mid<t}\left[4 \rho^{\prime} f|\langle A D v, \hat{x}\rangle|^{2}+2\left(f k_{2}+g \rho^{\prime}\right) \operatorname{Re}[\langle A D v, \hat{x}\rangle \bar{v}]\right. \\
& \left.+\left\{(g-\langle A \nabla f, \hat{x}\rangle-f \operatorname{div}(A \hat{x})) k_{1}-f\left\langle A \nabla k_{1}, \hat{x}\right\rangle+g k_{2}\right\}|v|^{2}\right] d x .
\end{aligned}
$$

Proof. $v(x) \in H_{\mathrm{loc}}^{2}\left(E_{R_{0}}\right)$ (, where $E_{R_{0}}=\left\{x|| x \mid>R_{0}\right\}$,) given in Definition 2.5 satisfies

$$
\begin{aligned}
- & \langle D, A D v\rangle+2 \rho^{\prime}(r)\langle A D v, \hat{x}\rangle \\
& +\left\{\left(q_{1}(x)+k_{1}(x)\right)+\left(q_{2}(x)+k_{2}(x)\right)\right\} v(x)=0
\end{aligned}
$$

for $r>R_{0}$. Noting (A1), (A2), (B1), (B2), (C1), (C2), (C3), (Dl) and (D2) we apply Lemma 2.7 to

$$
\begin{aligned}
0= & \operatorname{Re} \int_{s<\mid x_{i}<t}\left[-\langle D, A D v\rangle+2 \rho^{\prime}\langle A D v, \hat{x}\rangle+\left\{\left(q_{1}+k_{1}\right)+\left(q_{2}+k_{2}\right)\right\} v\right] \\
& \times[2 f(x)\langle A \overline{D v}, \hat{x}\rangle+g(x) \bar{v}] d x .
\end{aligned}
$$

At the same time we note

$$
\begin{aligned}
& D_{i} D_{l}-D_{l} D_{i}=\sqrt{-1}\left\{\partial_{i} b_{l}(x)-\partial_{l} b_{i}(x)\right\}, \\
& 2 \operatorname{Re}\left[\left\langle D_{l} D w, A \overline{D w}\right\rangle\right]=\partial_{l}(\langle A D w, \overline{D w}\rangle)-\left\langle\left(\partial_{l} A\right) D w, \overline{D w}\right\rangle, \\
& \langle A B A \hat{x}, \hat{x}\rangle=0 .
\end{aligned}
$$

Then we have the followings: 


$$
\begin{aligned}
& -2 \operatorname{Re} \int_{s<|x|<t}\langle D, A D v\rangle f\langle A \overline{D v}, \hat{x}\rangle d x \\
& =-2\left(\int_{|x|=t}-\int_{|x|=s}\right) f|\langle A D v, \hat{x}\rangle|^{2} d S \\
& +\int_{s<|x|<t}\left[2 \operatorname{Re}[\langle A D v, \nabla f\rangle\langle A \overline{D v}, \hat{x}\rangle]+2 r^{-1} f\left(|A D v|^{2}\right.\right. \\
& \left.-|\langle A D v, \hat{x}\rangle|^{2}\right)+2 f \operatorname{Re}[\langle(\langle A D v, \nabla\rangle A) \overline{D v}, \hat{x}\rangle] \\
& \left.+2 f\left\{\sum_{i, k, l=1}^{n}(A D v)_{i} \hat{x}_{k} a_{k l} \overline{D_{i} D_{l} v}\right\}\right] d x \text {, } \\
& 2 \operatorname{Re} \int_{s<|x|<t} f\left\{\sum_{i, k, l=1}^{n}(A D v)_{i} \hat{x}_{k} a_{k l} \overline{D_{i} D_{l} v}\right\} d x \\
& =\int_{s<|x|<t}[f\langle\hat{x}, A \nabla\rangle(\langle A D v, \overline{D v}\rangle)-f\langle(\langle\hat{x}, A D\rangle A) D v, \overline{D v}\rangle \\
& \left.-2 f \operatorname{Re}\left[\sqrt{-1}\left\langle A B A \hat{x},\left(D v-\hat{x}\langle A \hat{x}, \hat{x}\rangle^{-1}\langle A D v, \hat{x}\rangle\right)\right\rangle \bar{v}\right]\right] d x, \\
& \int_{s<|x|<t} f\langle\hat{x}, A D\rangle(\langle A D v, \overline{D v}\rangle) d x \\
& =\left(\int_{|x|=t}-\int_{|x|=s}\right) f\langle A \hat{x}, \hat{x}\rangle\langle A D v, \overline{D v}\rangle d S \\
& -\int_{s<|x|<t}\{\langle A \nabla f, \hat{x}\rangle+f \operatorname{div}(A \hat{x})\}\langle A D v, \overline{D v}\rangle d x, \\
& 2 \operatorname{Re} \int_{s<|x|<t}\left(q_{1}+k_{1}\right) v f\langle A \overline{D v}, \hat{x}\rangle d x \\
& =\left(\int_{|x|=t}-\int_{|x|=s}\right) f\langle A \hat{x}, \hat{x}\rangle\left(q_{1}+k_{1}\right)|v|^{2} d S \\
& -\int_{s<|x|<t}\left[\{\langle A \nabla f, \hat{x}\rangle+f \operatorname{div}(A \hat{x})\}\left(q_{1}+k_{1}\right)\right. \\
& \left.+f\left\langle A D\left(q_{1}+k_{1}\right), \hat{x}\right\rangle\right\}|v|^{2} d x,-\operatorname{Re} \int_{s<|x|<t}\langle D, A D v\rangle g \bar{v} d x \\
& =-\left(\int_{|x|=t}-\int_{|x|=s}\right) g \operatorname{Re}[\langle A D v, \hat{x}\rangle \bar{v}] d S \\
& +\int_{s<|x|<t}[\operatorname{Re}[\langle A D v, \nabla g\rangle \bar{v}]+g\langle A D v, \overline{D v}\rangle] d x \text {. }
\end{aligned}
$$

So we have the assertion.

Definition 2.9. Let

$$
\begin{aligned}
G(x ; v) & =(2 \alpha-\gamma(x))\langle A \hat{x}, \hat{x}\rangle^{-1}|\langle A D v, \hat{x}\rangle|^{2}+(2-\gamma(x))\{\langle A D v, \overline{D v}\rangle \\
- & \left.\langle A \hat{x}, \hat{x}\rangle^{-1}|\langle A D v, \hat{x}\rangle|^{2}\right\}+2 r \operatorname{Re}\left[\left\langle A D v, \nabla\left(\langle A \hat{x}, \hat{x}\rangle^{-1}\right)\right\rangle\langle A \overline{D v}, \hat{x}\rangle\right] \\
& +2\left\{\langle A \hat{x}, \hat{x}\rangle^{-1}|A D v|^{2}-\langle A D v, \overline{D v}\rangle\right\} \\
& +2 r\langle A \hat{x}, \hat{x}\rangle^{-1} \operatorname{Re}[\langle(\langle A D v, \nabla\rangle A) \overline{D v}, \hat{x}\rangle]
\end{aligned}
$$




$$
\begin{aligned}
& -r\langle A \hat{x}, \hat{x}\rangle^{-1} \operatorname{Re}[\langle(\langle\hat{x}, A D\rangle A) D v, \overline{D v}\rangle] \\
& -2\langle A \hat{x}, \hat{x}\rangle^{-1} \operatorname{Re}\left[\sqrt{-1}\left\langle A B A x,\left(D v-\hat{x}\langle A \hat{x}, \hat{x}\rangle^{-1}\langle A D v, \hat{x}\rangle\right)\right\rangle \bar{v}\right] \\
& +2 r\langle A \hat{x}, \hat{x}\rangle^{-1} \operatorname{Re}\left[\langle A D v, \hat{x}\rangle \overline{q_{2}}\right]+(\alpha-1) g_{0}(x) r^{-1} \operatorname{Re}[\langle A D v, \hat{x}\rangle \bar{v}] \\
& +\operatorname{Re}\left[\left\langle A D v, \nabla g_{0}\right\rangle \bar{v}\right]+\left\{g_{0}(x) \operatorname{Re}\left[q_{2}\right]-r\langle A \hat{x}, \hat{x}\rangle^{-1}\left\langle A V q_{1}, \hat{x}\right\rangle\right. \\
& \left.-\gamma(x) q_{1}\right\}|v|^{2},
\end{aligned}
$$

where

$$
g_{0}(x)=\alpha-\gamma(x)+r\langle A \hat{x}, \hat{x}\rangle^{-1} \operatorname{div}(A \hat{x})+r\left\langle A \nabla\left(\langle A \hat{x}, \hat{x}\rangle^{-1}\right), \hat{x}\right\rangle .
$$

The meanings of $G(x ; v)$ and $g_{0}(x)$ given in Definition 2.9 can be cleared by the following. The choice of $f(x)$ and $g(x)$ given in the following are the same given by Mochizuki [6].

Lemma 2.10. we have

(1) for $t>s>R_{0}$

$$
\begin{aligned}
F(t ; \rho, & \left.\langle A \hat{x}, \hat{x}\rangle^{-1} r^{\alpha}, g_{0}(x) r^{\alpha-1}\right)-F\left(s ; \rho,\langle A \hat{x}, \hat{x}\rangle^{-1} r^{\alpha}, g_{0}(x) r^{\alpha-1}\right) \\
= & \int_{s<|x|<t} r^{\alpha-1}\left[G(x ; v)+4 \rho^{\prime} r\langle A \hat{x}, \hat{x}\rangle^{-1}|\langle A D v, \hat{x}\rangle|^{2}\right. \\
& +2\left\{r \rho^{\prime \prime}+r \rho^{\prime}\langle A \hat{x}, \hat{x}\rangle^{-1} \operatorname{div}(A \hat{x})+g_{0} \rho^{\prime}\right\} \operatorname{Re}[\langle A D v, \hat{x}\rangle \bar{v}] \\
& +\langle A \hat{x}, \hat{x}\rangle\left\{r(x) \rho^{\prime 2}+2 r \rho^{\prime} \rho^{\prime \prime}-r \rho^{\prime 2}\langle A D(\langle A \hat{x}, \hat{x}\rangle-1), \hat{x}\rangle\right. \\
& \left.\left.+g_{0} \rho^{\prime \prime}+g_{0} \rho^{\prime}\langle A \hat{x}, \hat{x}\rangle^{-1} \operatorname{div}(A \hat{x})\right\}|v|^{2}\right] d x,
\end{aligned}
$$

(2) $g_{0}(x)=n+\alpha-1-\gamma(x)+o(1)$ as $\quad$ i $x \mid \rightarrow \infty$,

(3) $\nabla g_{0}(x)=o\left(r^{\beta-1}\right)$ as $|x| \rightarrow \infty$.

Proof. Noting Definitions 2.5, 2.9 and Lemma 2.8, we have (1) by direct calculation. (2) and (3) are also obtained by $\beta>0,(\mathrm{~A} 5)$, (F5), Lemma 2.1 and Definition 2.9.

Lemma 2.11. There exist some constants $C_{8}>0$ and $R_{2}>R_{0}$ such that for any $r>R_{2}$ we have

$$
G(x ; v) \geq C_{8}\left(|\langle A D v, \hat{x}\rangle|^{2}+r^{2 \beta-2}|v|^{2}\right)
$$

and for any $t>s>R_{2}$ we have

$$
\begin{aligned}
& F\left(t ; 0,\langle A \hat{x}, \hat{x}\rangle^{-1} r^{\alpha}, g_{0}(x) r^{\alpha-1}\right)-F\left(s ; 0,\langle A \hat{x}, \hat{x}\rangle^{-1} r^{\alpha}, g_{0}(x) r^{\alpha-1}\right) \\
& \geq C_{8} \int_{s<|x|<t} r^{\alpha-1}\left[|\langle A D v, \hat{x}\rangle|^{2}+r^{2 \beta-2}|v|^{2}\right] d x .
\end{aligned}
$$

Proof. Noting Lemma 2.10, we have only to show the former 
part of the assertion. In the sequel $\varepsilon_{i}(r)(i=1,2, \ldots)$ means a positive function for $r>R_{0}$ which tends to 0 as $r \rightarrow \infty$. Noting (Al) (A5), Lemmas 2.1, 2.2, and 2.10 and inequality

$|\langle A(x) \xi, \eta\rangle| \leq\langle A(x) \xi, \bar{\xi}\rangle^{1 / 2}\langle A(x) \eta, \bar{\eta}\rangle^{1 / 2} \quad$ for any $x \in \Omega, \xi, \eta \in \boldsymbol{C}^{n}$, we have the followings:

$$
\begin{aligned}
& 2 r \operatorname{Re}\left[\left\langle A D v, \nabla\left(\langle A \hat{x}, \hat{x}\rangle^{-1}\right)\right\rangle\langle A \overline{D v}, \hat{x}\rangle\right] \geq-\varepsilon_{1}(r)\langle A D v, \overline{D v}\rangle, \\
& 2\left\{\langle A \hat{x}, \hat{x}\rangle^{-1}|A D v|^{2}-\langle A D v, \overline{D v}\rangle\right] \geq-\varepsilon_{2}(r)\langle A D v, \overline{D v}\rangle, \\
& 2 r\langle A \hat{x}, \hat{x}\rangle^{-1} \operatorname{Re}[\langle(\langle A D v, \nabla\rangle A) \overline{D v}, \hat{x}\rangle] \geq-\varepsilon_{3}(r)\langle A D v, \overline{D v}\rangle, \\
& -r\langle A \hat{x}, \hat{x}\rangle^{-1} \operatorname{Re}[\langle(\langle\hat{x}, A \nabla\rangle A) D v, \overline{D v}\rangle] \geq-\varepsilon_{4}(r)\langle A D v, \overline{D v}\rangle, \\
& -2\langle A \hat{x}, \hat{x}\rangle^{-1} \operatorname{Re}\left[\sqrt{-1}\left\langle A B A x,\left(D v-\hat{x}\langle A \hat{x}, \hat{x}\rangle^{-1}\langle A D v, \hat{x}\rangle\right)\right\rangle \overline{]}\right] \\
& \geq-2|v|\langle A \hat{x}, \hat{x}\rangle^{-1}\langle A B A x, B A x\rangle^{1 / 2}\left\langle A\left(D v-\hat{x}(A \hat{x}, \hat{x}\rangle^{-1}\langle A D v, \hat{x}\rangle\right),\right. \\
& \left.\quad\left(\overline{D v}-\hat{x}\langle A \hat{x}, \hat{x}\rangle^{-1}\langle A \overline{D v}, \hat{x}\rangle\right)\right\rangle^{1 / 2} \\
& \geq-\frac{2-\gamma(x)}{1+\eta}\left\{\langle A D v, \overline{D v}\rangle-\langle A \hat{x}, \hat{x}\rangle^{-1}|\langle A D v, \hat{x}\rangle|^{2}\right\} \\
& \quad-\frac{1+\eta}{2-\gamma(x)}\langle A \hat{x}, \hat{x}\rangle^{-2}\langle A B A x, B A x\rangle|v|^{2}, \\
& 2 r\langle A \hat{x}, \hat{x}\rangle^{-1} \operatorname{Re}\left[\langle A D v, \hat{x}\rangle \overline{q_{2} v}\right] \geq-\frac{2 \alpha-\gamma(x)}{1+(\eta / 2)}\langle A \hat{x}, \hat{x}\rangle^{-1}|\langle A D v, \hat{x}\rangle|^{2} \\
& \quad-\frac{1+(\eta / 2)}{2 \alpha-\gamma(x)}\langle A \hat{x}, \hat{x}\rangle^{-1}\left|r q_{2}\right|^{2}|v|^{2}, \\
& (\alpha-1) g_{0} r^{-1} \operatorname{Re}[\langle A D v, \hat{x}\rangle \bar{v}] \geq-r^{-\beta}\langle A \hat{x}, \hat{x}\rangle^{-1}|\langle A D v, \hat{x}\rangle|^{2} \\
& \quad-4^{-1}(\alpha-1)^{2}\left|g_{0}\right|^{2} r^{\beta-2}\langle A \hat{x}, \hat{x}\rangle|v|^{2}, \\
& \operatorname{Re}\left[\left\langle A D v, \nabla g_{0}\right\rangle \bar{v}\right] \geq-\varepsilon_{5}(r)\left\{\langle A D v, \overline{D v}\rangle+r^{2 \beta-2}|v|^{2}\right\}, \\
& g_{0} \operatorname{Re}\left[q_{2}\right] \geq-\frac{\eta}{2(2 \alpha-\gamma(x))}\langle A \hat{x}, \hat{x}\rangle^{-1}\left|r q_{2}\right|^{2}-\frac{2 \alpha-\gamma(x)}{2 \eta}\langle A \hat{x}, \hat{x}\rangle\left|g_{0}\right|^{2} r^{-2} .
\end{aligned}
$$

By (F), (F1), (F2), (F3), and Lemmas 2.1 and 2.10, there exist some constants $C_{8}>0$ and $R_{2}>R_{0}$ such that for any $r>R_{2}$ we have

$$
\begin{aligned}
& 2 \alpha-\gamma(x)-\frac{2 \alpha-\gamma(x)}{1+(\eta / 2)}-r^{-\beta}-\sum_{i=1}^{5} \varepsilon_{i}(r) \geq C_{1} C_{8}, \\
& 2-\gamma(x)-\frac{2-\gamma(x)}{1+\eta}-\sum_{i=1}^{5} \varepsilon_{i}(r) \geq C_{1} C_{8}, \\
& -\left[r\langle A \hat{x}, \hat{x}\rangle^{-1}\left\langle A \nabla q_{1}, \hat{x}\right\rangle+\gamma(x) q_{1}\right.
\end{aligned}
$$




$$
\begin{aligned}
+ & (1+\eta)\left\{\frac{1}{2 \alpha-\gamma(x)}\langle A \hat{x}, \hat{x}\rangle^{-1}\left|r q_{2}\right|^{2}\right. \\
& \left.\left.+\frac{1}{2-\gamma(x)}\langle A \hat{x}, \hat{x}\rangle^{-2}\langle A B A x, B A x\rangle\right\}\right] \\
& -4^{-1}(\alpha-1)^{2}\left|g_{0}\right|^{2}\langle A \hat{x}, \hat{x}\rangle r^{\beta-2}-\varepsilon_{5}(r) r^{2 \beta-2} \\
& -\frac{2 \alpha-\gamma(x)}{2 \eta}\langle A \hat{x}, \hat{x}\rangle\left|g_{0}\right|^{2} r^{-2} \geq C_{8} r^{2 \beta-2},
\end{aligned}
$$

where $C_{1}$ is the one given in (A4). So, by Definition 2.9 and $\angle A D v$, $\overline{D v}\rangle=\langle A \hat{x}, \hat{x}\rangle^{-1}|\langle A D v, \hat{x}\rangle|^{2}+\left\{\langle A D v, \overline{D v}\rangle-\langle A \hat{x}, \hat{x}\rangle^{-1}|\langle A D v, \hat{x}\rangle|^{2}\right\}$, we have the assertion.

Remark 2.12. Under the assumptions in Theorem 1.1 we have also $G(x ; v) \geq C_{8}\left(|D v|^{2}+r^{2 \beta-2}|v|^{2}\right)$. But considering the proof of Theorem 1.4, we formalize the statement given in Lemma 2.11.

Lemma 2. 13. For any constant $l>2^{-1}(2-\lim$ inf $\gamma(x)) \geq \delta / 2>0$, there exists some constant $R_{3} \geq R_{2}$ (, where $R_{2}$ is the one given in Lemma 2.11 ,) such that for any $t>s>R_{3}$ and any $m \geq 1$ we have

$$
F\left(t ; m r^{l},\langle A \hat{x}, \hat{x}\rangle^{-1} r^{\alpha}, g_{0}(x) r^{\alpha-1}\right) \geq F\left(s ; m r^{l},\langle A \hat{x}, \hat{x}\rangle^{-1} r^{\alpha}, g_{0}(x) r^{\alpha-1}\right) .
$$

Proof. Let

$$
\rho(r)=m r^{l}, \quad f(x)=\langle A \hat{x}, \hat{x}\rangle^{-1} r^{\alpha}, \quad g(x)=g_{0}(x) r^{\alpha-1} .
$$

Then we have

$$
\begin{aligned}
& 4 \rho^{\prime} r\langle A \hat{x}, \hat{x}\rangle^{-1}|\langle A D v, \hat{x}\rangle|^{2}=4 m l r^{l}\langle A \hat{x}, \hat{x}\rangle^{-1}|\langle A D v, \hat{x}\rangle|^{2}, \\
& 2\left(r \rho^{\prime \prime}+r \rho^{\prime}\langle A \hat{x}, \hat{x}\rangle^{-1} \operatorname{div}(A \hat{x})+g_{0} \rho^{\prime}\right) \operatorname{Re}[\langle A D v, \hat{x}\rangle \bar{v}] \\
&=2 \mathrm{~m} l r^{l-1}\left\{l-1+r\langle A \hat{x}, \hat{x}\rangle^{-1} \operatorname{div}(A \hat{x})+g_{0}\right\} \operatorname{Re}[\langle A D v, \hat{x}\rangle \bar{v}] \\
& \geq-m l r^{l}\langle A \hat{x}, \hat{x}\rangle^{-1}|\langle A D v, \hat{x}\rangle|^{2} \\
& \quad-m l r^{1-2}\langle A \hat{x}, \hat{x}\rangle\left\{l-1+r\langle A \hat{x}, \hat{x}\rangle^{-1} \operatorname{div}(A \hat{x})+g_{0}\right\}^{2}|v|^{2}, \\
& r \rho^{\prime 2}+2 r \rho^{\prime} \rho^{\prime \prime}-r \rho^{\prime 2}\left\langle A D\left(\langle A \hat{x}, \hat{x}\rangle^{-1}\right), \hat{x}\right\rangle+g_{0} \rho^{\prime \prime}+g_{0} \rho^{\prime}\langle A \hat{x}, \hat{x}\rangle^{-1} \operatorname{div}(A \hat{x}) \\
&= m^{2} l^{2} r^{2 l-2}\left\{2 l+r(x)-2-r\left\langle A D\left(\langle A \hat{x}, \hat{x}\rangle^{-1}\right), \hat{x}\right\rangle\right\} \\
&+g_{0} m l r^{l-2}\left\{l-1+r\langle A \hat{x}, \hat{x}\rangle^{-1} \operatorname{div}(A \hat{x})\right\} .
\end{aligned}
$$

By Lemmas 2.1 and 2.10, there exists some constant $R_{3} \geq R_{2}$ such that for any $r>R_{3}$ and any $m \geq 1$ we have

$$
m l r^{l}\left\{2 l+\gamma(x)-2-r\left\langle A D\left(\langle A \hat{x}, \hat{x}\rangle^{-1}\right), \hat{x}\right\rangle\right\}
$$




$$
\begin{aligned}
& +g_{0}\left\{l-1+r\langle A \hat{x}, \hat{x}\rangle^{-1} \operatorname{div}(A \hat{x})\right\} \\
& \quad-\langle A \hat{x}, \hat{x}\rangle\left\{l-1+r\langle A \hat{x}, \hat{x}\rangle^{-1} \operatorname{div}(A \hat{x})+g_{0}\right\}^{2} \geq 0 .
\end{aligned}
$$

So, by Lemmas 2.10 and 2.11, we have the assertion.

\section{§ 3. Proofs of Theorem 1.1 and Corollary 1.2}

In this section we assume Assumptions $(\mathrm{A}) \sim(\mathrm{G})$. And let $u(x)$ satisfy $\left(^{*}\right)$. Keep in our mind that we intend to prove Theorem 1.1 by contradiction.

Lemma 3.1. If

$$
\liminf _{R \rightarrow \infty} R^{\alpha} \int_{|x|=R}\left[|\langle A D u, \hat{x}\rangle|^{2}+\left\{r^{-2}+\left(q_{1}\right)_{-}\right\}|u|^{2}\right] d x=0,
$$

then we have for any $m>0$

$$
\begin{gathered}
\int_{|x|>R_{0}} r^{m}\left[|D u|^{2}+\left\{1+\left(q_{1}\right)_{-}\right\}|u|^{2}\right] d x<\infty, \\
\lim _{R \rightarrow \infty} \inf ^{m} \int_{|x|=R}\left[|D u|^{2}+\left\{1+\left(q_{1}\right)_{-}\right\}|u|^{2}\right] d S=0 .
\end{gathered}
$$

Proof. We have only to prove the former part of the assertion. At first we show

$$
\left(^{\dagger}\right): \int_{|x|>R_{0}}\left[r^{m \beta+\alpha-1}|\langle A D u, \hat{x}\rangle|^{2}+r^{(m+2) \beta+\alpha-3}|u|^{2}\right] d x<\infty
$$

for $m=0,1,2, \ldots$, by mathematical induction. By Definition 2.5 and Lemmas 2.1 and 2.10, there exists some constant $C_{9}>0$ such that for any $t \geq R_{0}$ we have

$$
\begin{aligned}
F(t ; 0, & \left.\langle A \hat{x}, \hat{x}\rangle^{-1} r^{\alpha}, g_{0}(x) r^{\alpha-1}\right) \\
= & t^{\alpha} \int_{|x|=t}\left[2\langle A \hat{x}, \hat{x}\rangle^{-1}|\langle A D u, \hat{x}\rangle|^{2}-\langle A D u, \overline{D u}\rangle\right. \\
& \left.+g_{0} r^{-1} \operatorname{Re}[\langle A D u, \hat{x}\rangle \bar{u}]-q_{1}|u|^{2}\right] d S \\
& \leq C_{9} t^{\alpha} \int_{|x|=t}\left[|\langle A D u, \hat{x}\rangle|^{2}+\left\{r^{-2}+\left(q_{1}\right)_{-}\right\}|u|^{2}\right] d S .
\end{aligned}
$$

Then by assumption we have

$$
\lim _{t \rightarrow \infty} \inf _{t \rightarrow \infty} F\left(t ; 0,\langle A \hat{x}, \hat{x}\rangle^{-1} r^{\alpha}, g_{0}(x) r^{\alpha-1}\right) \leq 0 .
$$


Therefore, letting $t \rightarrow \infty$ along the suitable subsequence in Lemma 2. 11, we have for any $s \geq R_{2}$

$$
C_{8} \int_{|x|>s}\left[r^{\alpha-1}|\langle A D u, \hat{x}\rangle|^{2}+r^{\alpha+2 \beta-3}|u|^{2}\right] d x \leq-F\left(s ; 0,\langle A \hat{x}, \hat{x}\rangle^{-1} r^{\alpha}, g_{0} r^{\alpha-1}\right),
$$

which shows that $\left(^{\dagger}\right)$ holds for $m=0$. Now we assume that $\left({ }^{\dagger}\right)$ holds for $m$. Then by above inequality, Definition 2.5 and Lemma 2.8 we have for any $t \geq R_{2}$

$$
\begin{aligned}
& C_{8} \int_{R_{2}}^{t} s^{(m+1) \beta-1} d s \int_{|x|>s}\left[r^{\alpha-1}|\langle A D u, \hat{x}\rangle|^{2}+r^{\alpha+2 \beta-3}|u|^{2}\right] d x \\
& \leq-\int_{R_{2}}^{t} s^{(m+1) \beta-1} F\left(s ; 0,\langle A \hat{x}, \hat{x}\rangle^{-1} r^{\alpha}, g_{0} r^{\alpha-1}\right) d s \\
& =\int_{R_{2}<|x|<t} r^{(m+1) \beta+\alpha-1}\left[\langle A D u, \overline{D u}\rangle+q_{1}|u|^{2}-g_{0} r^{-1} \operatorname{Re}[\langle A D u, \hat{x}\rangle \bar{u}]\right. \\
& \left.\quad-2\langle A \hat{x}, \hat{x}\rangle^{-1}|\langle A D u, \hat{x}\rangle|^{2}\right] d x \\
& =F\left(t ; 0,0, r^{(m+1) \beta+\alpha-1}\right)-F\left(R_{2} ; 0 ; 0, r^{(m+1) \beta+\alpha-1}\right) \\
& \quad-\int_{R_{2}<|x|<t} r^{(m+1) \beta+\alpha-1}\left[2\langle A \hat{x}, \hat{x}\rangle^{-1}|\langle A D u, \hat{x}\rangle|^{2}\right. \\
& \left.\quad+\left\{g_{0}+(m+1) \beta+\alpha-1\right\} r^{-1} \operatorname{Re}[\langle A D u, \hat{x}\rangle \bar{u}]+\operatorname{Re}\left[q_{2}\right]|u|^{2}\right] d x .
\end{aligned}
$$

Noting $\beta>0$, (G2), Lemmas 2.1 and 2.10, and $\left(^{\dagger}\right)$ for $m$, there exists some constant $C_{10}>0$ such that for any $t>R_{2}$ we have

$$
\begin{aligned}
& \quad-\int_{R_{2}<|x|<t} r^{(m+1) \beta+\alpha-1}\left[2\langle A \hat{x}, \hat{x}\rangle^{-1}|\langle A D u, \hat{x}\rangle|^{2}\right. \\
& \left.\quad+\left\{g_{0}+(m+1) \beta+\alpha-1\right\} r^{-1} \operatorname{Re}[\langle A D u, \hat{x}\rangle \bar{u}]+\operatorname{Re}\left[q_{2}\right]|u|^{2}\right] d x \\
& \leq \int_{|x|>\mid R_{2}} r^{(m+1) \beta+\alpha-1}\left[8^{-1}\langle A \hat{x}, \hat{x}\rangle\left\{g_{0}+(m+1) \beta+\alpha-1\right\}^{2} r^{-2}+C_{3} r^{\beta-2}\right]|u|^{2} d x \\
& \leq C_{10} \int_{|x|>R_{2}} r^{(m+2) \beta+\alpha-3}|u|^{2} d x<\infty .
\end{aligned}
$$

By Definition 2.5. we have

$$
\begin{aligned}
& F\left(t ; 0,0, r^{(m+1) \beta+\alpha-1}\right) \\
& \leq 2^{-1} \int_{|x|=t}\left[r^{m \beta+\alpha}|\langle A D u, \hat{x}\rangle|^{2}+r^{(m+2) \beta+\alpha-2}|u|^{2}\right] d s .
\end{aligned}
$$

Then by $(\dagger)$ for $m$, we have

$$
\lim _{t \rightarrow \infty} \inf F\left(t ; 0,0, r^{(m+1) \beta+\alpha-1}\right) \leq 0 .
$$

Therefore 


$$
\begin{aligned}
& +\infty\rangle \int_{R_{2}}^{\infty} s^{(m+1) \beta-1} d s \int_{|x|>s}\left[r^{\alpha-1}|\langle A D u, \hat{x}\rangle|^{2}+r^{\alpha+2 \beta-3}|u|^{2}\right] d x \\
& =\frac{1}{(m+1) \beta} \int_{|x|>R_{2}}\left(r^{(m+1) \beta}-R_{2}^{(m+1) \beta}\right)\left[r^{\alpha-1}|\langle A D u, \hat{x}\rangle|^{2}+r^{\alpha+2 \beta-3}|u|^{2}\right] d x,
\end{aligned}
$$

which shows that $(\dagger)$ holds for $m+1$. Letting in Lemma $2.4 \zeta(r)$ $=r^{m / 2}$, and noting $\left(^{\dagger}\right)$, we have the former half of the assertion.

\section{Lemma 3. 2. Let}

$$
\lim _{R \rightarrow \infty} \inf ^{\alpha} \int_{|x|=R}\left[|\langle A D u, \hat{x}\rangle|^{2}+\left\{r^{-2}+\left(q_{1}\right)_{-}\right\}|u|^{2}\right] d x=0 .
$$

Then for any $0<l<\beta$ and any $m>0$ there exists some constant $R_{4} \geq R_{2}$ (, where $R_{2}$ is the one given in Lemma 2.11) such that for any $t>s>$ $R_{4}$ we have

$$
e^{m t^{2}} \int_{|x|=t}\langle A \hat{x}, \hat{x}\rangle|u|^{2} d S \leq e^{m s} \int_{|x|=s}\langle A \hat{x}, \hat{x}\rangle|u|^{2} d S .
$$

Proof. For $0<l<\beta$ and $m>0$ let $a(r)=2^{-1}\left(m l r^{l}+n\right)$. By Lemma 2.1 there exists some constant $R_{5} \geq R_{0}$ such that for any $r \geq R_{5}$ we have $n \geq r\langle A \hat{x}, \hat{x}\rangle^{-1} \operatorname{div}(A \hat{x})$. Then for any $t>s>R_{5}$ we have by integration by parts

$$
\begin{aligned}
\left(\int_{|x|=t}\right. & \left.-\int_{|x|=s}\right) e^{m r^{l}}\langle A \hat{x}, \hat{x}\rangle|u|^{2} d S \\
& =\int_{s<|x|<t} e^{m r^{l}}\left[2 \operatorname{Re}[\langle A D u, \hat{x}\rangle \bar{u}]+\left\{m l r^{l-1}\langle A \hat{x}, \hat{x}\rangle+\operatorname{div}(A \hat{x})\right\}|u|^{2}\right] d x \\
& \leq 2 \int_{s}^{t} e^{m \tau^{l}} d \tau \int_{|x|=\tau}\left[\operatorname{Re}[\langle A D u, \hat{x}\rangle \bar{u}]+r^{-1} a(r)\langle A \hat{x}, \hat{x}\rangle|u|^{2}\right] d S .
\end{aligned}
$$

So we have only to show that there exists some $R_{4} \geq R_{5}$ such that for any $\tau>R_{4}$ we have

$$
\int_{|x|=\tau}\left[\operatorname{Re}[\langle A D u, \hat{x}\rangle \bar{u}]+r^{-1} a(r)\langle A \hat{x}, \hat{x}\rangle|u|^{2}\right] d S \leq 0 .
$$

For $\rho(r)=a(\tau) \log r$ and $g(r)=r^{-2 a(\tau)}$ we have $e^{2 \rho(r)} g(r)=1, e^{2 \rho(r)} g(r) \rho^{\prime}(r)$ $=r^{-1} a(\tau), \quad g^{\prime}(r)+2 g(r) \rho^{\prime}(r)=0, \quad k_{1}(x)=-a(\tau)^{2} r^{-2}\langle A \hat{x}, \hat{x}\rangle$ and $k_{2}(x)=$ $-a(\tau) r^{-2}\langle A \hat{x}, \hat{x}\rangle+a(\tau) r^{-1} \operatorname{div}(A \hat{x})$ by Definition 2.5. Therefore by Lemmas 2.6 and 2.8 we have for $t_{1}>\tau>R_{5}$

$$
\left(\int_{|x|=t_{1}}-\int_{|x|=\tau}\right)\left[\operatorname{Re}[\langle A D u, \hat{x}\rangle \bar{u}]+r^{-1} a(\tau)\langle A \hat{x}, \hat{x}\rangle|u|^{2}\right] d S
$$




$$
\begin{aligned}
= & F\left(t_{1} ; a(\tau) \log r, 0, r^{-2 a(\tau)}\right)-F\left(\tau ; a(\tau) \log \mathrm{r}, 0, r^{-2 a(\tau)}\right) \\
= & \int_{\tau<|x|<t_{1}} r^{-2 a(\tau)}\left[\langle A D v, \overline{D v}\rangle+\left\{q_{1}+\operatorname{Re}\left[q_{2}\right]\right\}|v|^{2}\right. \\
& \left.+a(\tau) r^{-2}\{r \operatorname{div}(A \hat{x})-\langle A \hat{x}, \hat{x}\rangle-a(\tau)\langle A \hat{x}, \hat{x}\rangle\}|v|^{2}\right] d x \\
= & -\int_{\tau}^{t_{1}} s_{1}^{-(a+2 a(\tau))}\left\{F\left(s_{1} ; a(\tau) \log r,\langle A \hat{x}, \hat{x}\rangle^{-1} r^{\alpha}, g_{0} r^{\alpha-1}\right)\right. \\
& \left.+2 C_{3} s_{1}^{\alpha+\beta-2} \int_{|x|=s_{1}}\langle A \hat{x}, \hat{x}\rangle|v|^{2} d S\right\} d s_{1} \\
& +\int_{\tau<|x|<t_{1}} r^{-2 a(\tau)}\left[2\langle A \hat{x}, \hat{x}\rangle^{-1}|\langle A D v, \hat{x}\rangle|^{2}+g_{0} r^{-1} \operatorname{Re}[\langle A D v, \hat{x}\rangle \bar{v}]\right. \\
& +\left\{\operatorname{Re}\left[q_{2}\right]+a(\tau) r^{-2}(r \operatorname{div}(A \hat{x})-\langle A \hat{x}, \hat{x}\rangle)\right. \\
& \left.\left.+2 C_{3} r^{\beta-2}\langle A \hat{x}, \hat{x}\rangle\right\}|v|^{2}\right] d x,
\end{aligned}
$$

where $C_{3}$ is the one given in (G2). By (G2), Lemmas 2.1 and 2. 10, and $0<l<\beta$, there exist some constants $R_{6} \geq R_{5}$ and $C_{11}>0$ such that for any $r>\tau \geq R_{6}$ we have

$$
\begin{aligned}
& 2\langle A \hat{x}, \hat{x}\rangle^{-1}|\langle A D v, \hat{x}\rangle|^{2}+g_{0} r^{-1} \operatorname{Re}[\langle A D v, \hat{x}\rangle \bar{v}] \\
& +\left\{\operatorname{Re}\left[q_{2}\right]+a(\tau) r^{-2}(r \operatorname{div}(A \hat{x})-\langle A \hat{x}, \hat{x}\rangle)+2 C_{3} r^{\beta-2}\langle A \hat{x}, \hat{x}\rangle\right\}|v|^{2} \\
& \geq\left\{-8^{-1} g_{0}^{2} r^{-2}\langle A \hat{x}, \hat{x}\rangle+2 C_{3}\langle A \hat{x}, \hat{x}\rangle r^{\beta-2}-\left(\operatorname{Re}\left[q_{2}\right]\right)-\right. \\
& \left.+a(\tau) r^{-2}(r \operatorname{div}(A \hat{x})-\langle A \hat{x}, \hat{x}\rangle)\right\}|v|^{2} \\
& \geq\left(2^{-1} C_{3} r^{\beta-2}-C_{11} r^{l-2}\right)|v|^{2} \geq 0 \text { 。 }
\end{aligned}
$$

Noting for $t_{2}>s_{1}>R_{6}$

$$
\begin{aligned}
\left(\int_{|x|=t_{2}}\right. & \left.-\int_{|x|=s_{1}}\right) r^{\alpha+\beta-2}\langle A \hat{x}, \hat{x}\rangle|v|^{2} d S \\
= & \int_{s_{1}<|x|<t_{2}} r^{\alpha+\beta-2}[2 \operatorname{Re}[\langle A D v, \hat{x}\rangle \bar{v}]+\{\operatorname{div}(A \hat{x}) \\
& \left.\left.+(\alpha+\beta-2)\langle A \hat{x}, \hat{x}\rangle r^{-1}\right\}|v|^{2}\right] d x,
\end{aligned}
$$

we have by Lemma 2.10 for $t_{1}, t_{2}>s_{1}>\tau>R_{6}$

$$
\begin{aligned}
& F\left(t_{2} ; a(\tau) \log r,\langle A \hat{x}, \hat{x}\rangle^{-1} r^{\alpha}, g_{0} r^{\alpha-1}\right)-F\left(s_{1} ; a(\tau) \log r,\langle A \hat{x}, \hat{x}\rangle^{-1} r^{\alpha}, g_{0} r^{\alpha-1}\right) \\
& +2 C_{3}\left(\int_{|x|=t_{2}}-\int_{|x|=s_{1}}\right) r^{\alpha+\beta-2}\langle A \hat{x}, \hat{x}\rangle|v|^{2} d S \\
& =\int_{s_{1}<|x|<t_{2}} r^{\alpha-1}\left[G(x ; v)+4 a(\tau)\langle A \hat{x}, \hat{x}\rangle^{-1}|\langle A D v, \hat{x}\rangle|^{2}\right. \\
& \quad+2 a(\tau) r^{-1}\left\{g_{0}+r\langle A \hat{x}, \hat{x}\rangle^{-1} \operatorname{div}(A \hat{x})-1\right\} \operatorname{Re}[\langle A D v, \hat{x}\rangle \bar{v}] \\
& \quad+a(\tau)\langle A \hat{x}, \hat{x}\rangle r^{-2}\left\{\left(\gamma(x)-2-r\left\langle A D\left(\langle A \hat{x}, \hat{x}\rangle^{-1}\right), \hat{x}\right\rangle\right) a(\tau)\right. \\
& \left.\quad-g_{0}+r g_{0}\langle A \hat{x}, \hat{x}\rangle^{-1} \operatorname{div}(A \hat{x})\right\}|v|^{2}+4 C_{3} r^{\beta-1} \operatorname{Re}[\langle A D v, \hat{x}\rangle \bar{v}] \\
& \left.\quad+2 C_{3}\langle A \hat{x}, \hat{x}\rangle\left\{r\langle A \hat{x}, \hat{x}\rangle^{-1} \operatorname{div}(A \hat{x})+\alpha+\beta-2\right\} r^{\beta-2}|v|^{2}\right] d x .
\end{aligned}
$$


Noting $0<l<\beta$, Lemmas 2.1,2.10 and 2.11, there exist some constants $R_{7} \geq \max \left\{R_{2}, R_{6}\right\}$ and $C_{12}>0$ such that for any $r>\tau>R_{7}$ we have

$$
\begin{aligned}
& G(x ; v) \geq C_{8} r^{2 \beta-2}|v|^{2}, \\
& 2 a(\tau) r^{-1}\left\{g_{0}+r\langle A \hat{x}, \hat{x}\rangle^{-1} \operatorname{div}(A \hat{x})-1\right\} \operatorname{Re}[\langle A D v, \hat{x}\rangle \bar{v}] \\
& \geq-a(\tau)\langle A \hat{x}, \hat{x}\rangle^{-1}|\langle A D v, \hat{x}\rangle|^{2}-C_{12} r^{l-2}|v|^{2}, \\
& a(\tau)\langle A \hat{x}, \hat{x}\rangle r^{-2}\left\{\left(\gamma-2-r\left\langle A D\left(\langle A \hat{x}, \hat{x}\rangle^{-1}\right), \hat{x}\right\rangle\right) a(\tau)\right. \\
& \left.\quad \quad-g_{0}+r g_{0}\langle A \hat{x}, \hat{x}\rangle^{-1} \operatorname{div}(A \hat{x})\right\}|v|^{2} \\
& \geq-C_{12} r^{2 l-2}|v|^{2}, \\
& 2 C_{3}\langle A \hat{x}, \hat{x}\rangle\left\{r\langle A \hat{x}, \hat{x}\rangle^{-1} \operatorname{div}(A \hat{x})+\alpha+\beta-2\right\} r^{\beta-2}|v|^{2} \geq-C_{12} r^{\beta-2}|v|^{2}, \\
& 4 C_{3} r^{\beta-1} \operatorname{Re}[\langle A D v, \hat{x}\rangle \bar{v}] \geq-a(\tau)\langle A \hat{x}, \hat{x}\rangle^{-1}|\langle A D v, \hat{x}\rangle|^{2}-C_{12} r^{2 \beta-2} \tau^{-l}|v|^{2} .
\end{aligned}
$$

There exists some constant $R_{4}>R_{7} \geq R_{2}$ such that for any $r>\tau>R_{4}$ we have

$$
C_{8} r^{2 \beta-2}-C_{12} 2^{2^{\beta-2} \tau^{-l}}-C_{12}\left(r^{l-2}+r^{2 l-2}+r^{\beta-2}\right)>0 .
$$

Therefore for any $t_{2}>s_{1}>\tau>R_{4}$ we have

$$
\begin{aligned}
& F\left(t_{2} ; a(\tau) \log r,\langle A \hat{x}, \hat{x}\rangle^{-1} r^{\alpha}, g_{0} r^{\alpha-1}\right)+2 C_{3} \int_{|x|=t_{2}} r^{\alpha+\beta-2}\langle A \hat{x}, \hat{x}\rangle|v|^{2} d S \\
& \geq F\left(s_{1} ; a(\tau) \log r,\langle A \hat{x}, \hat{x}\rangle^{-1} r^{\alpha}, g_{0} r^{\alpha-1}\right)+2 C_{3} \int_{|x|=s_{1}} r^{\alpha+\beta-2}\langle A \hat{x}, \hat{x}\rangle|v|^{2} d S .
\end{aligned}
$$

By Lemmas 2.1, 2.6 and 2.10, for $\tau \geq R_{4}$ there exist some constant $R_{8} \geq R_{4}$ and $C_{13}>0$ such that for any $t_{2} \geq R_{8}$ we have

$$
\begin{aligned}
& F\left(t_{2} ; a(\tau) \log \mathrm{r},\langle A \hat{x}, \hat{x}\rangle^{-1} r^{\alpha}, g_{0} r^{\alpha-1}\right)+2 C_{3} \int_{|x|=t_{2}} r^{\alpha+\beta-2}\langle A \hat{x}, \hat{x}\rangle|v|^{2} d S \\
& \leq C_{13} t_{2}^{\alpha+2 a(\tau)} \int_{|x|=t_{2}}\left[|\langle A D u, \hat{x}\rangle|^{2}+\left\{r^{\beta-2}+\left(q_{1}\right)_{-}\right\}|u|^{2}\right] d S .
\end{aligned}
$$

Therefore by Lemma 3.1 we have

$$
\begin{aligned}
& \liminf _{t_{2} \rightarrow \infty}\left[F\left(t_{2} ; a(\tau) \log r,\langle A \hat{x}, \hat{x}\rangle^{-1} r^{\alpha}, g_{0} r^{\alpha-1}\right)\right. \\
& \left.\quad+2 C_{3} \int_{|x|=t_{2}} r^{\alpha+\beta-2}\langle A \hat{x}, \hat{x}\rangle|v|^{2} d S\right] \leq 0 .
\end{aligned}
$$

So for any $s_{1} \geq \tau \geq R_{4}$ we have

$$
F\left(s_{1} ; a(\tau) \log r,\langle A \hat{x}, \hat{x}\rangle^{-1} r^{\alpha}, g_{0} r^{\alpha-1}\right)+2 C_{3} \int_{|x|=s_{1}} r^{\alpha+\beta-2}\langle A \hat{x}, \hat{x}\rangle|v|^{2} d S \leq 0,
$$


and then for any $t_{1} \geq \tau \geq R_{4}$ we have

$$
\begin{aligned}
& \int_{|x|=t_{1}}\left[\operatorname{Re}[\langle A D u, \hat{x}\rangle \bar{u}]+a(\tau) r^{-1}\langle A \hat{x}, \hat{x}\rangle|u|^{2}\right] d S \\
& \geq \int_{|x|=\tau}\left[\operatorname{Re}[\langle A D u, \hat{x}\rangle \bar{u}]+a(\tau) r^{-1}\langle A \hat{x}, \hat{x}\rangle|u|^{2}\right] d S .
\end{aligned}
$$

Letting $t_{1} \rightarrow \infty$ along the suitable subsequence, we have, by Lemma 3.1., for any $\tau \geq R_{4}$

$$
\int_{|x|=\tau}\left[\operatorname{Re}[\langle A D u, \hat{x}\rangle \bar{u}]+a(\tau) r^{-1}\langle A \hat{x}, \hat{x}\rangle|u|^{2}\right] d S \leq 0 .
$$

Lemma 3.3. If

$$
\lim _{R \rightarrow \infty} \inf R^{\alpha} \int_{|x|=R}\left[|\langle A D u, \hat{x}\rangle|^{2}+\left\{r^{-2}+\left(q_{1}\right)_{-}\right\}|u|^{2}\right] d S=0,
$$

then for any $0<l<\beta$ and any $m>0$ we have

$$
\int_{|x|>R_{0}} e^{m r^{l}}\left[|D u|^{2}+\left\{1+\left(q_{1}\right)_{-}\right\}|u|^{2}\right] d x<\infty
$$

Proof. We apply Lemma 3.2 replacing $m$ wfth $m+2$. Then for any $r \geq R_{4}$ we have

$$
\int_{|x|=r}|u|^{2} d S \leq C_{14} e^{-(m+2) r^{l}}
$$

where $C_{14}=e^{(m+2) R_{4}^{l}} \int_{|x|=R_{4}}|u|^{2} d S \geq 0$. Then we have

$$
\int_{|x| \geq R_{0}} e^{(m+1) r}|u|^{2} d x \leq e^{(m+1) R 4} \int_{R_{0}<|x|<R_{4}}|u|^{2} d x+C_{14} \int_{R_{4}}^{\infty} e^{-r^{l}} d r<\infty .
$$

Letting $\zeta(r)=e^{(m / 2) r^{l}}$ in Lemma 2.4, we have the assertion.

Now we can prove Theorem 1.1.

Proof of Theorem 1.1. At first we shall prove the former half of the statement. If we assume that the statement is false, we have

$$
\liminf _{R \rightarrow \infty} R^{\alpha} \int_{|x|=R}\left[|\langle A D u, \hat{x}\rangle|^{2}+\left\{r^{-2}+\left(q_{1}\right)_{-}\right\}|u|^{2}\right] d S=0 .
$$

By (F) and (F2) we can choose a constant $l$ satisfying $0<2^{-1} \delta \leq$ $2^{-1}\left(2-\liminf _{r \rightarrow \infty} \gamma(x)\right)<l<\beta$. By Lemma 2.12 we have for any $t>s \geq$ 
$R_{3}$ and any $m \geq 1$

$$
F\left(t ; m r^{l},\langle A \hat{x}, \hat{x}\rangle^{-1} r^{\alpha}, g_{0} r^{\alpha-1}\right) \geq F\left(s ; m r^{l},\langle A \hat{x}, \hat{x}\rangle^{-1} r^{\alpha}, g_{0} r^{\alpha-1}\right) .
$$

By Lemmas 2.1, 2.6 and 2.10, for $m \geq 1$ there exists some constant $R_{9} \geq R_{3}$ such that for any $t \geq R_{9}$ we have

$$
\begin{aligned}
& F\left(t ; m r^{l},\langle A \hat{x}, \hat{x}\rangle^{-1} r^{\alpha}, g_{0} r^{\alpha-1}\right) \\
& \leq e^{(2 m+1) t^{l}} \int_{|x|=t}\left[\left.\langle A D u, \hat{x}\rangle\right|^{2}+\left\{1+\left(q_{1}\right)_{-}\right\}|u|^{2}\right] d S .
\end{aligned}
$$

So by Lemma 3.3 we have

$$
\lim _{t \rightarrow \infty} \inf F\left(t ; m r^{l},\langle A \hat{x}, \hat{x}\rangle^{-1} r^{\alpha}, g_{0} r^{\alpha-1}\right) \leq 0,
$$

and then we have for any $s \geq R_{3}$ and any $m \geq 1$

$$
F\left(s ; m r^{l},\langle A \hat{x}, \hat{x}\rangle^{-1} r^{\alpha}, g_{0} r^{\alpha-1}\right) \leq 0 .
$$

Since $\operatorname{supp}[u]$ is not a compact set in $\bar{\Omega}$ by $\left(^{*}\right)$, there exists some constant $R_{10} \geq R_{3}$ such that $\int_{|x|=R_{10}}\langle A \hat{x}, \hat{x}\rangle|u|^{2} d S>0$. By Lemma 2.6, $e^{-2 m R_{10}^{l} F\left(R_{10} ; m r^{l},\langle A \hat{x}, \hat{x}\rangle^{-1} r^{\alpha}, g_{0} r^{\alpha-1}\right)}$ is a quadratic in $m$, whose coefficient of $m^{2}$ is $2 l^{2} R_{10}^{2 l+\alpha-2} \int_{|x|=R_{10}}\langle A \hat{x}, \hat{x}\rangle|u|^{2} d S>0$. Therefore there exists some constant $m_{0} \geq 1$ such that

$$
F\left(R_{10} ; m_{0} r^{l},\langle A \hat{x}, \hat{x}\rangle^{-1} r^{\alpha}, g_{0} r^{\alpha-1}\right)>0,
$$

which is the contradiction.

Now we shall prove the latter half of Theorem 1.1. Let $\varepsilon>0$. We take $\xi_{R}(r) \in C_{0}^{1}(R, R+\varepsilon)$ satisfying $\xi_{R}(r)=1$ for $R+(\varepsilon / 3) \leq r \leq R$ $+(2 \varepsilon / 3)$ and $0 \leq \xi_{R}(r) \leq 1$ for $R \leq r \leq R+\varepsilon$. Moreover we can admit that there exists some constant $C_{15}>0$ such that for any $R \geq R_{0}$ and any $r \geq R$ we have $\left|\xi_{R}^{\prime}(r)\right| \leq C_{15}$. Let $\sigma=\max \left\{0, \delta_{1}, \delta_{2}\right\} \geq 0$. By the conclusion of the former half of Theorem 1.1, there exist some constants $C_{16}>0$ and $R_{11} \geq R_{0}$ such that for any $R \geq R_{11}$ we have

$$
\begin{aligned}
& C_{16} R^{-\alpha-\sigma} \leq \int_{R+(\varepsilon / 3)}^{R+(2 \varepsilon / 3)} r^{-\alpha-\sigma} d r, \\
& C_{16} R^{-\alpha} \leq \int_{|x|=R}\left[|\langle A D u, \hat{x}\rangle|^{2}+\left\{r^{-2}+\left(q_{1}\right)_{-}\right\}|u|^{2}\right] d S .
\end{aligned}
$$

Then by (A4), Lemmas 2.2, 2.3, $-\sigma \leq 0$ and $-\sigma+\max \left\{\delta_{1}, \delta_{2}\right\} \leq 0$, we have for any $R \geq R_{11}$ 


$$
\begin{aligned}
C_{16}^{2} R^{-\alpha-\sigma} \leq & \int_{R+(\varepsilon / 3)}^{R+(2 \varepsilon / 3)} C_{16} r^{-\alpha-\sigma} d r \\
\leq & \int_{R+(\varepsilon / 3)<|x|<R+(2 \varepsilon / 3)} r^{-\sigma}\left[|\langle A D u, \hat{x}\rangle|^{2}+\left\{r^{-2}+\left(q_{1}\right)-\right\}|u|^{2}\right] d x \\
\leq & C_{1}^{2} \int_{R<|x|<R+\varepsilon}\left(\xi_{R}(r) r^{-\sigma / 2}\right)^{2}\left[|D u|^{2}+\left(q_{1}\right)-|u|^{2}\right] d x \\
& +\int_{R<|x|<R+\varepsilon} r^{-\sigma-2}|u|^{2} d x \\
\leq & C_{1}^{2} C_{4} \int_{R<|x|<R+\varepsilon}\left\{r^{\left.\max \mid \delta_{1}, \delta_{2}\right\}} \xi_{R}^{2} r^{-\sigma}+2 \xi_{R}^{\prime 2} r^{-\sigma}\right. \\
& \left.+2(-\sigma / 2)^{2} \xi_{R}^{2} r^{-\sigma-2}\right\}|u|^{2} d x+\int_{R<|x|<R+\varepsilon} r^{-\sigma-2}|u|^{2} d x \\
\leq & C_{17} \int_{R<|x|<R+\varepsilon}|u|^{2} d x
\end{aligned}
$$

where $C_{1}$ is the one given in (A4) and $C_{17}=C_{1}^{2} C_{4}\left(1+2 C_{15}^{2}+\frac{\sigma^{2}}{2}\right)+1>$ 0 。

Proof of Corollary 1.2. By Theorem 1.1 there exist some constant $C_{18}>0$ and some integer $N_{0} \geq R_{0}$ such that for any integer $N \geq$ $N_{0}$ we have

$$
\int_{N<|x|<N+1}|u|^{2} d x \geq C_{18} N^{-\alpha-\max \left\{0, \delta_{1}, \delta_{2}\right\}} \geq C_{18} N^{-1}
$$

Then we have

$$
\int_{N_{0}<|x|<N+1}|u|^{2} d x=\sum_{n=N_{0}}^{N} \int_{n<|x|<n+1}|u|^{2} d x \geq C_{18} \sum_{n=N_{0}}^{N} n^{-1},
$$

and then $\int_{|x| \geq R_{0}}|u|^{2} d x=\infty$.

\section{\$4. Proofs of Theorems 1.3 and 1.4}

In this section we assume $(\mathrm{B}) \sim(\mathrm{G})$ replacing $a_{i j}(x)$ with $\delta_{i j}$ except for (C3). Instead of (C3) we assume (C3)'. Let $u(x)$ satisfy (**). In this case we use the following.

Lemma 4. 1. Let $w, \partial_{r} w \in L_{\mathrm{loc}}^{1}(\Omega)$, where $\partial_{r} w$ is the derivative in the distribution sense. Then for any $t>R_{0}, \int_{|x|=t} w d S$ exists (by choosing suitable representative for $w$, if necessary), and for any $t>s>R_{0}$ we have 


$$
\int_{s<|x|<t} \partial_{r} w d x=\left(\int_{|x|=t} \int_{|x|=s}\right) w d S-\int_{s<|x|<t}(n-1) r^{-1} w d x 。
$$

Proof. See Lemma 4.1 of Uchiyama [11].

Remark 4.2. If $a_{i j}(x)=\delta_{i j}$ and if we assume (C3)' instead of (C3), then Lemma 2.8 with $a_{i j}(x)=\delta_{i j}$ also holds. In fact by (C3)' we have $f\left(q_{1}+k_{1}\right)|v|^{2}, \partial_{r}\left\{f\left(q_{1}+k_{1}\right)|v|^{2}\right\} \in L_{\mathrm{loc}}^{1}(\Omega)$, and then by Lemma 4. 1 we have

$$
\begin{aligned}
2 \operatorname{Re} \int_{s<|x|<t}\left(q_{1}+k_{1}\right) v f\langle\overline{D v}, \hat{x}\rangle d x \\
=\left(\int_{|x|=t}-\int_{|x|=s}\right) f\left(q_{1}+k_{1}\right)|v|^{2} d S \\
\quad-\int_{s<|x|<t}\left[\left\{\partial_{r} f+(n-1) r^{-1} f\right\}\left(q_{1}+k_{1}\right)+f \partial_{r}\left(q_{1}+k_{1}\right)\right]|v|^{2} d x .
\end{aligned}
$$

Now we can prove Theorems 1.3 and 1.4.

Proof of Theorem 1.3. Since Lemma 2.8 with $a_{i j}(x)=\delta_{i j}$ is also true by Remark 4.2, we can follow the proofs of Theorem 1.1 and Corollary 1.2.

Proof of Theorem 1.4. Since Lemma 2.8 with $a_{i j}(x)=\delta_{i j}$ and $b_{i}(x)=0$ is also true by Remark 4.2, we have for $t>s>R_{0}$ by Definition 2.9 and Lemma 2.10

$$
\begin{aligned}
& F\left(t ; \rho, r^{\alpha}, g_{0} r^{\alpha-1}\right)-F\left(s ; \rho, r^{\alpha}, g_{0} r^{\alpha-1}\right) \\
& \quad=\int_{s<|x|<t} r^{\alpha-1}\left[G(x ; v)+4 \rho^{\prime} r\left|\partial_{r} v\right|^{2}+2\left\{r \rho^{\prime \prime}+(n-1) \rho^{\prime}+g_{0} \rho^{\prime}\right\} \operatorname{Re}\left[\bar{v} \partial_{r} v\right]\right. \\
& \left.\quad+\left\{\gamma(x) \rho^{\prime 2}+2 r \rho^{\prime} \rho^{\prime \prime}+g_{0} \rho^{\prime \prime}+(n-1) g_{0} \rho^{\prime} r^{-1}\right\}|v|^{2}\right] d x,
\end{aligned}
$$

where

$$
\begin{aligned}
g_{0}(x)=\alpha & +n-1-\gamma(x)=O(1) \quad \text { as }|x| \rightarrow \infty, \\
G(x ; v)= & (2 \alpha-\gamma(x))\left|\partial_{r} v\right|^{2}+(2-\gamma(x))\left(|\nabla v|^{2}-\left|\partial_{r} v\right|^{2}\right)+2 r \operatorname{Re}\left[\left(\partial_{r} v\right) \overline{q_{2} v}\right] \\
& +(\alpha-1) g_{0} r^{-1} \operatorname{Re}\left[\bar{v} \partial_{r} v\right]+\operatorname{Re}\left[\left\langle\nabla v, \nabla g_{0}\right\rangle \bar{v}\right] \\
& +\left\{g_{0} \operatorname{Re}\left[q_{2}\right]-r \partial_{r} q_{1}-\gamma(x) q_{1}\right\}|v|^{2} .
\end{aligned}
$$

By (F2)' and (F5)' we have

$$
\left\langle\nabla v, \nabla g_{0}\right\rangle=\left(\partial_{r} g_{0}\right) \partial_{r} v+\left\langle\left(\nabla-\hat{x} \partial_{r}\right) v,\left(\nabla-\hat{x} \partial_{r}\right) g_{0}\right\rangle,
$$




$$
\begin{aligned}
& \partial_{r} g_{0}=o\left(r^{\beta-1}\right) \quad \text { as }|x| \rightarrow \infty, \\
& \left|\left(\nabla-\hat{x} \partial_{r}\right) g_{0}\right|=\left|\left(\nabla-\hat{x} \partial_{r}\right) \gamma(x)\right| \leq(2-\gamma(x))^{1 / 2} p(x) \quad \text { for } r>R_{0}, \\
& p(x)=o\left(r^{\beta-1}\right) \quad \text { as }|x| \rightarrow \infty .
\end{aligned}
$$

So we have

$$
\begin{aligned}
& 2 r \operatorname{Re}\left[\left(\partial_{r} v\right) \overline{q_{2} v}\right] \geq-\frac{2 \alpha-\gamma(x)}{1+(\eta / 2)}\left|\partial_{r} v\right|^{2}-\frac{1+(\eta / 2)}{2 \alpha-\gamma(x)}\left|r q_{2}\right|^{2}|v|^{2}, \\
& (\alpha-1) g_{0} r^{-1} \operatorname{Re}\left[\bar{v} \partial_{r} v\right] \geq-r^{-\beta}\left|\partial_{r} v\right|^{2}-4^{-1}(\alpha-1)^{2}\left|g_{0}\right|^{2} r^{\beta-2}|v|^{2}, \\
& \operatorname{Re}\left[\left\langle\nabla v, \nabla g_{0}\right\rangle \bar{v}\right] \geq-\varepsilon_{6}(r)\left\{\left|\partial_{r} v\right|^{2}+r^{2 \beta-2}|v|^{2}\right\}-(2-\gamma(x))\left\{|\nabla v|^{2}-\left|\partial_{r} v\right|^{2}\right\}, \\
& g_{0} \operatorname{Re}\left[q_{2}\right] \geq-\frac{\eta}{2(2 \alpha-\gamma(x)}\left|r q_{2}\right|^{2}-\frac{2 \alpha-\gamma(x)}{2 \eta}\left|g_{0}\right|^{2} r^{-2} .
\end{aligned}
$$

By (F), (F1) and (F3), there exist some constant $C_{19}>0$ and $R_{12}>$ $R_{0}$ such that for any $r>R_{12}$ we have

$$
\begin{aligned}
2 \alpha-\gamma(x)- & \frac{2 \alpha-\gamma(x)}{1+(\eta / 2)}-r^{-\beta}-\varepsilon_{6}(r) \geq C_{19}, \\
- & \left\{r \partial_{r} q_{1}+\gamma(x) q_{1}+\frac{1+\eta}{2 \alpha-\gamma(x)}\left|r q_{2}\right|^{2}\right\}-4^{-1}(\alpha-1)^{2}\left|g_{0}\right|^{2} r^{\beta-2} \\
& -\varepsilon_{6}(r) r^{2 \beta-2}-\frac{2 \alpha-\gamma(x)}{2 \eta}\left|g_{0}\right|^{2} r^{-2} \\
\geq & C_{19} r^{2 \beta-2} .
\end{aligned}
$$

So for any $r \geq R_{12}$ we have

$$
G(x ; v) \geq C_{19}\left\{\left|\partial_{r} v\right|^{2}+r^{2 \beta-2}|v|^{2}\right\},
$$

which corresponds to Lemma 2.11. Therefore we can follow the proofs of Theorem 1.1 and Corollary 1.2.

\section{§5. Examples}

In this section we give several applications of Theorem 1.3. Let $u(x)$ satisfy $\left({ }^{*}\right)$, where $\left({ }^{* *}\right)$ is given in Theorem 1.3. In the sequel we treat special $b_{i}(x), q_{1}(x)$ and $q_{2}(x)$ in (**). So it is easy to check that all assumptions (B), (C)', (D) and (E) are satisfied, where (C)' is the condition given by a replacement (C3) with (C3)' in (G). So we are mainly interested in checking (F) and (G). In (F) we choose $\gamma(x)$ as a constant function, and we rewrite its constant as $\gamma$ again. After checking that (F) and (G) hold, we have by Theorem 1.3 


$$
\text { (\#) } \lim _{R \rightarrow \infty} \inf ^{\mu} \int_{R<|x|<R+1}|u(x)|^{2} d x>0
$$

where

$$
\mu=\alpha+\max \left\{0, \delta_{1}, \delta_{2}\right\},
$$

and $\alpha, \delta_{1}, \delta_{2}$ are the ones given in (F) and (G). Here we note that the choice of $\alpha$ in $(\mathrm{F})$ has some freedom. The smaller we choose $\alpha$, the better results for $\mu$ we have.

Example 5. 1. In (**) let

$$
\begin{aligned}
& q_{1}(x)=-h(x)+V(x)-\lambda, \\
& q_{2}(x)=o\left(r^{(\theta / 2)-1}\right) \quad \text { as } r \rightarrow \infty, \\
& b_{i}(x)=0,
\end{aligned}
$$

where $h(x)$ is a positive continuous homogeneous function of degree $\theta>0, V(x)$ is a real-valued function satisfying

$$
\text { (\#\#) } V(x)=o\left(r^{\theta}\right) \text { and } \partial_{r} V(x)=o\left(r^{\theta-1}\right) \text { as } r \rightarrow \infty \text {, }
$$

and $\lambda$ is a real constant.

Note $r \partial_{r} q_{1}+\gamma q_{1} \leq-\{\theta+\gamma-o(1)\} r^{\theta} \min _{|x|=1} h(x)$. So $\beta=2^{-1}(2+\theta)>0$ and $\min \{2,2 \alpha\}>\gamma>2-2 \beta=-\theta$ satisfy $(\mathrm{F}) . \quad \delta_{1}=\theta>\delta_{2}=(\theta / 2)-1=\beta-2$ satisfy (G). Then in (\#) $\mu>\theta / 2$ is arbitrary. Moreover if $0<\theta$ $<2$, we have $u(x) \notin L^{2}(\Omega)$. Compare with Agmon [2, Theorem 5] and Uchiyama [8, Example 5.2].

Example 5. 2. In (**) let

$$
\begin{aligned}
& q_{1}(x)=-h(x)+V(x), \\
& q_{2}(x)=o\left(r^{(\theta / 2)-1}\right) \quad \text { as } r \rightarrow \infty, \\
& b_{i}(x)=0,
\end{aligned}
$$

where $h(x)$ is a positive continuous homogeneous function of degree $-2<\theta<0$ and $V(x)$ is a real-valued function satisfying (\#\#).

In this case $\beta=2^{-1}(2+\theta)>0$ and $\min \{2,2 \alpha\}>\gamma>2-2 \beta=-\theta$ satisfy (F), and $0>\delta_{1}=\theta>\delta_{2}=(\theta / 2)-1=\beta-2$ satisfy (G). Then in (\#) $\mu>$ $-(\theta / 2)$ is arbitrary and $u(x) \notin L^{2}(\Omega)$. Compare with Agmon [1, Theorem 4 without its proof] and Uchiyama [8, Example 5.2]. 
Example 5. 3. In (**) let

$$
\begin{aligned}
& q_{1}(x)=h_{1}(x)-h_{2}(x)+V(x)-\lambda, \\
& q_{2}(x)=o\left(r^{0}\right) \quad \text { as } r \rightarrow \infty, \\
& b_{i}(x)=0,
\end{aligned}
$$

where $h_{1}(x)$ is a real-valued homogeneous function of degree $-\gamma>$ -2 satisfying

(\#\#\#) there exist some constant $0<a<1$ and $C>0$ such that for any $w(x) \in C_{0}^{\infty}(\Omega)$ we have

$$
\int_{\Omega}\left|h_{1}(x)\right||w(x)|^{2} d x \leq a \int_{\Omega}|\nabla w(x)|^{2} d x+C \int_{\Omega} r^{\max \{0,-r\}}|w(x)|^{2} d x,
$$

$h_{2}(x)$ is a real-valued continuous homogeneous function of degree $\theta$, $V(x)$ is a real-valued function satisfying (\#\#), and $\lambda$ is a real constant. Here we do not assume that $h_{1}(x)$ has a constant sign.

If $h_{2}(x)$ is positive, $\theta>\max \{0,-\gamma\}$ and $\sigma=(\theta / 2)-1$, then in (\#) $\mu>(\gamma / 2)+\theta$ is arbitrary, since $\beta=2^{-1}(2+\theta)$ and $2 \alpha>\gamma>2-2 \beta=-\theta$ satisfy $(F)$ and $\delta_{1}=\theta>\delta_{2}=(\theta / 2)-1=\beta-2$ satisfy (G). So if $h_{2}(x)$ is positive, $-2<\gamma<2, \max \{0,-\gamma\}<\theta<1-(\gamma / 2)$ and $\sigma=(\theta / 2)-1$, then $u(x) \notin L^{2}(\Omega)$. On the other hand if $\gamma>0, \lambda>0, \theta<0$ and $\sigma=$ -1 , then in (\#) $\mu>\gamma / 2$ is arbitrary and $u(x) \notin L^{2}(\Omega)$, since $\beta=1$, $2 \alpha>\gamma>2-2 \beta=0, \delta_{1}=0$ and $\delta_{2}=-1=\beta-2$ satisfy $(\mathbb{F})$ and $(\mathrm{G})$. Compare with Uchiyama [8, Example 5.6 and Remark 5.3].

Remark 5.4. We note that

$$
h_{1}(x)=-\sum_{1 \leq i \leq N} \frac{c_{i}}{r_{i}}+\sum_{1 \leq i<j \leq N} \frac{c_{i j}}{r_{i j}}
$$

is a homogeneous function of degree -1 , does not have a constant sign and satisfies the condition (\#\#\#) given in Example 5.3, where $c_{i}, c_{i j}$ are positive constants, $n=3 N, x=\left(x^{(1)}, \ldots, x^{(N)}\right) \in \mathbb{R}^{3 N}, x^{(i)} \in \mathbb{R}^{3}$, $r_{i}=\left|x^{(i)}\right|$ and $r_{i j}=\left|x^{(i)}-x^{(j)}\right|$.

Example 5.5. In (**) let $n=2$ and

$$
\begin{aligned}
& q_{1}(x)=c r^{\theta}+V(x)-\lambda, \\
& q_{2}(x)=o\left(r^{0}\right) \quad \text { as } r \rightarrow \infty, \\
& b_{1}(x)=-\frac{1}{2} b_{0} x_{2} f(r), \quad b_{2}(x)=\frac{1}{2} b_{0} x_{1} f(r),
\end{aligned}
$$


where $V(x)$ is a real-valued function satisfying (\#\#), $f(r)=r^{\delta}$ or $f(r)$ $=(1+r)^{\delta}, b_{0} \neq 0, c, \lambda, \theta, \sigma, \delta$ are real constants.

Noting

$$
|B(x) x|^{2}=\frac{1}{4} b_{0}^{2}\left\{r f^{\prime}(r)+2 f(r)\right\}^{2} r^{2}
$$

we have the followings:

(i) If $c<0, \theta>\max \{0,2(\delta+1)\}$ and $\sigma=(\theta / 2)-1$, then in (\#) $\mu>\theta / 2$ is arbitrary, since $\beta=2^{-1}(2+\theta)>0,2>\gamma>2-2 \beta=-\theta$ and $\alpha$ $>\gamma / 2$ satisfy $(\mathrm{F})$, and $\delta_{1}=\theta>\delta_{2}=\sigma=(\theta / 2)-1=\beta-2$ satisfy (G). So if $c<0,2>\theta>\max \{0,2(\delta+1)\}$ and $\sigma=(\theta / 2)-1$, then $u(x) \notin L^{2}(\Omega)$.

(ii) If $\theta=2(\delta+1)>0, \sigma=(\theta / 2)-1$ and $b_{0}^{2}+4 c<0$, then in (\#) $\mu>\frac{1}{4}\left[2-\theta-(2+\theta)\left\{1+b_{0}^{2}(4 c)^{-1}\right\}^{1 / 2}\right]+\theta$ is arbitrary. In fact for any $\gamma$ satisfying $(2-2 \beta=-\theta<) \frac{1}{2}\left[2-\theta-(2+\theta)\left\{1+b_{0}^{2}(4 c)^{-1}\right\}^{1 / 2}\right]<\gamma<\frac{1}{2}[2$ $\left.-\theta+(2+\theta)\left\{1+b_{0}^{2}(4 c)^{-1}\right\}^{1 / 2}\right](<2)$, we have $c(\theta+\gamma)+\frac{1}{2-\gamma} \frac{1}{4} b_{0}^{2}(\delta+$ $2)^{2}<0$, which shows that $\beta=2^{-1}(2+\theta), \gamma$ given above and $2 \alpha>\gamma$ satisfy (F). $\delta_{1}=\theta>\delta_{2}=(\theta / 2)-1=\beta-2$ satisfy (G). So if $0<\theta=2(\delta+1)<$ $2\left[1+\left\{1+b_{0}^{2}(4 c)^{-1}\right\}^{1 / 2}\right]\left[3-\left\{1+b_{0}^{2}(4 c)^{-1}\right\}^{1 / 2}\right]^{-1}$ and $\sigma=(\theta / 2)-1$, then $u(x)$ $\notin L^{2}(\Omega)$.

(iii) If $\theta<0, \sigma=\delta=-1$ and $4 \lambda>b_{0}^{2}$, then in (\#) $\mu>\frac{1}{2}[1-\{1-$ $\left.\left.b_{0}^{2}(4 \lambda)^{-1}\right\}^{1 / 2}\right]$ is arbitrary and $u(x) \notin L^{2}(\Omega)$. In fact for any $\gamma$ satisfying $(2-2 \beta=0<) \quad 1-\left\{1-b_{0}^{2}(4 \lambda)^{-1}\right\}^{1 / 2}<\gamma<1+\left\{1-b_{0}^{2}(4 \lambda)^{-1}\right\}^{1 / 2}(<2)$, we have $-\gamma \lambda+\frac{1}{2-\gamma} \frac{1}{4} b_{0}^{2}<0$, which appears in $(\mathrm{F}) .(\mathrm{F})$ is satisfied by $\beta=1$, $\gamma$ given above and $2 \alpha>\gamma$. (G) is satisfied by $\delta_{1}=0$ and $\delta_{2}=-1=$ $\beta-2$.

(iv) If $\theta<0, \delta<-1, \sigma=-1$ and $\lambda>0$, then in (\#) $\mu>0$ is arbitrary and $u(x) \notin L^{2}(\Omega)$. In fact $\beta=1,0<\gamma<2$ and $2 \alpha>\gamma$ satisfy (F) and $\delta_{1}=0$ and $\delta_{2}=-1=\beta-2$ satisfy (G). Compare with Uchiyama [10, Example 7].

Example 5. 6. In (**) let $n=3$ and

$$
\begin{aligned}
& q_{1}(x)=c r^{\theta}+V(x)-\lambda, \\
& q_{2}(x)=o\left(r^{\sigma}\right) \quad \text { as } r \rightarrow \infty, \\
& b_{1}(x)=-\frac{1}{2} b_{0} x_{2} f(x), b_{2}(x)=\frac{1}{2} b_{0} x_{1} f(x), b_{3}(x)=0,
\end{aligned}
$$


where $f(x)=r^{\delta}\left(x_{1}^{2}+x_{2}^{2}\right)^{\delta^{\prime} / 2}, b_{0} \neq 0, \delta^{\prime}>-1, \delta, c, \theta, \sigma$ are real constants and $V(x)$ is a real-valued function satisfying (\#\#). The condition $\delta^{\prime}>-1$ is imposed to satisfy (B2).

Noting

$$
|B(x) x|^{2}=\frac{1}{4} b_{0}^{2}\left(x_{1}^{2}+x_{2}^{2}\right)\left(r \partial_{r} f+2 f\right)^{2}=\frac{1}{4} b_{0}^{2}\left(\delta+\delta^{\prime}+2\right)^{2} r^{2 \delta}\left(x_{1}^{2}+x_{2}^{2}\right)^{\delta^{\prime}+1},
$$

we have the same results as Example 5.5 (i) (iv), where we replace $\delta$ with $\delta+\delta^{\prime}$. Compare with Uchiyama [10, Example 10 ].

Example 5. 7. Let $N \geq 1$ be integer, $c_{i}, c_{\imath j}, c, b_{0} \neq 0, \delta, \delta^{\prime}, \theta, \sigma$ and $\lambda$ be real constants. And in (**) let $n=3 N$ and

$$
\begin{aligned}
& q_{1}(x)=-\sum_{1 \leq \imath \leq N} \frac{c_{i}}{r_{i}}+\sum_{1 \leq \imath<j \leq N} \frac{c_{\imath j}}{r_{\imath j}}+c r^{\theta}+V(x)-\lambda, \\
& q_{2}(x)=o\left(r^{\sigma}\right) \quad \text { as } r \rightarrow \infty, \\
& b_{3 i-2}(x)=-\frac{1}{2} b_{0} x_{3 \imath-1} f_{\imath}(x), b_{3 \imath-1}(x)=\frac{1}{2} b_{0} x_{3 t-2} f_{i}(x), b_{3 i}(x)=0,
\end{aligned}
$$

where $f_{\imath}(x)=r^{\delta} r_{\imath}^{\delta^{\prime}}\left(\delta^{\prime} \geq-1\right)$ or $f_{1}(x)=r^{\delta}\left(x_{3 \imath-2}^{2}+x_{3 \imath-1}^{2}\right)^{\delta^{\prime} / 2}\left(\delta^{\prime}>-1\right)$ and $V(x)$ is a real-valued function satisfying (\#\#).

Since

$$
\begin{aligned}
& \max \left\{s_{1}^{\tau}+\cdots+s_{N}^{\tau} \mid s_{1}^{2}+\cdots+s_{N}^{2}=1, s_{1} \geq 0,1 \leq i \leq N\right\} \\
& =\max \left\{N^{1-(\tau / 2)}, 1\right\} \quad \text { for } \tau \geq 0,
\end{aligned}
$$

we have

$$
\begin{array}{r}
|B(x) x|^{2}=\frac{1}{4} b_{0}^{2} \sum_{1 \leq \imath \leq N}\left(x_{3 \imath-2}^{2}+x_{3 \imath-1}^{2}\right)\left(r \partial_{r} f_{\imath}+2 f_{\imath}\right)^{2} \\
=\frac{1}{4} b_{0}^{2}\left(\delta+\delta^{\prime}+2\right)^{2} \sum_{1 \leq i \leq N}\left(x_{3 \imath-2}^{2}+x_{3 \imath-1}^{2}\right) f_{\imath}^{2}, \\
\limsup _{r \rightarrow \infty} r^{-2\left(\delta^{\prime}+1\right)} \sum_{1 \leq i \leq N}\left(x_{3 i-2}^{2}+x_{3 i-1}^{2}\right) r_{\imath}^{2 \delta^{\prime}}=\max \left\{N^{-\delta^{\prime}}, 1\right\}, \\
\limsup _{r \rightarrow \infty} r^{-2\left(\delta^{\prime}+1\right)} \sum_{1 \leq \imath \leq N}\left(x_{3 i-2}^{2}+x_{3 i-1}^{2}\right)^{\delta^{\prime}+1}=\max \left\{N^{-\delta}, 1\right\} .
\end{array}
$$

Noting Remark, 5.4, we must choose $2-2 \beta<\gamma=1<2$ in (F), and we have the followings:

(i) If $c<0, \theta>\max \left\{0,2\left(\delta+\delta^{\prime}+1\right)\right\}$ and $\sigma=(\theta / 2)-1$, then in (\#) $\mu>\frac{1}{2}+\theta$ is arbitrary. In fact $\beta=2^{-1}(2+\theta)>0$ and $\min \{2,2 \alpha\}>\gamma=$ $1>2-2 \beta=-\theta$ satisfy $(\mathrm{F})$, and $\delta_{1}=\theta>\delta_{2}=\sigma=\beta-2$ satisfy (G). So if 
$c<0, \frac{1}{2}>\theta>\max \left\{0,2\left(\delta+\delta^{\prime}+1\right)\right\}$ and $\sigma=(\theta / 2)-1$, then $u(x) \notin L^{2}(\Omega)$.

(ii) If $\theta=2\left(\delta+\delta^{\prime}+1\right)>0, \quad \sigma=(\theta / 2)-1 \quad$ and $16 c(\theta+1)+$ $b_{0}^{2}(\theta+2)^{2} \max \left\{N^{-\delta^{\prime}}, 1\right\}<0$, then in (\#) $\mu>\frac{1}{2}+\theta$ is arbitrary. In fact the choice of $\alpha, \beta, \gamma, \delta_{1}, \delta_{2}$ given in (i) also satisfies (F) and (G) in this case. So if $\frac{1}{2}>\theta=2\left(\delta+\delta^{\prime}+1\right)>0, \sigma=(\theta / 2)-1$ and $16 c(\theta+1)+$ $b_{0}^{2}(\theta+2)^{2} \max \left\{N^{-\delta^{\prime}}, 1\right\}<0$, then $u(x) \notin L^{2}(\Omega)$.

(iii) If $\theta<0, \delta+\delta^{\prime}=\sigma=-1$ and $4 \lambda>b_{0}^{2} \max \left\{N^{-\delta^{\prime}}, 1\right\}$, then in (\#) $\mu>1 / 2$ is arbitrary and $u(x) \notin L^{2}(\Omega)$, since $\beta=1,2 \alpha>\gamma=1>2-2 \beta$ $=0$ satisfy $(\mathrm{F})$ and $\delta_{1}=0>\delta_{2}=\sigma=-1=\beta-2$ satisfy $(\mathrm{G})$.

(iv) If $\theta<0, \delta+\delta^{\prime}<-1, \sigma=-1$ and $\lambda=0$, then in (\#) $\mu>1 / 2$ is arbitrary and $u(x) \notin L^{2}(\Omega)$, since $\beta=1,2 \alpha>\gamma=1>2-2 \beta=0$ satisfy (F), and $\delta_{1}=0$ and $\delta_{2}=\sigma=-1=\beta-2$ satisfy (G). Compare with Eastham-Kalf [3, Example 5.3.3, p. 134] and Uchiyama [10, Example $11]$.

Example 5. 8. In $(* *)$ let

$$
\begin{aligned}
& q_{1}(x)+q_{2}(x)=\frac{b \sin 2 a r}{r}+V(x)+o\left(r^{-1}\right)-\lambda \text { as } r \rightarrow \infty, \\
& b_{i}(x)=0,
\end{aligned}
$$

where $a>0, b \neq 0$ and $\lambda>0$ are real constants, and real-valued function $V(x)$ satisfies (\#\#) with $\theta=0$.

We shall show that $u(x) \notin L^{2}(\Omega)$, if $\lambda>2^{-1} a\left[\left\{a^{2}+4 b^{2}\right\}^{1 / 2}-a\right]$. For simplicity let $b= \pm \tau a(\tau>0)$ and $\lambda=\nu a^{2}(\nu>0)$. And let

$$
\begin{aligned}
& q_{1}(x)= \pm \tau a(1-s) \frac{\sin 2 a r}{r}+V(x)-\nu a^{2}, \\
& q_{2}(x)= \pm \tau a s \frac{\sin 2 a r}{r}+o\left(r^{-1}\right) \text { as } r \rightarrow \infty,
\end{aligned}
$$

where $-\infty<s<\infty$ is a parameter. Let $\alpha=\beta=1, \delta_{1}=0, \delta_{2}=-1=$ $\beta-2$ and $2-2 \beta=0<\gamma=2-t<2 \alpha=2(0<t<2)$, which satisfy (G). If (F) holds for some $-\infty<s_{0}<\infty$ and $0<t_{0}<2$, then $\mu=1$ satisfy (\#) and $u(x) \notin L^{2}(\Omega)$. In order to show that (F) holds for some $-\infty$ $<s_{0}<\infty$ and $0<t_{0}<2$, it is enough to show

$$
\inf \{H(s, t) \mid-\infty<s<\infty, 0<t<2\}<0,
$$

where 


$$
H(s, t)=a^{-2} \lim _{r \rightarrow \infty} \sup \left[r \partial_{r} q_{1}(x)+(2-t) q_{1}(x)+t^{-1}\left|r q_{2}(x)\right|^{2}\right] .
$$

Now' we calculate $H(s, t)$ as follows.

$$
\begin{aligned}
H(s, t)= & \limsup _{r \rightarrow \infty}\left[\tau^{2} s^{2} t^{-1}+\nu t-\tau^{2} s^{2} t^{-1}\left\{\cos 2 a r \mp(1-s) t \tau^{-1} s^{-2}\right\}^{2}\right. \\
& \left.+t(1-s)^{2} s^{-2}-2 \nu\right] \\
= & \begin{cases}\tau^{2} s^{2} t^{-1}+\nu t+t(1-s)^{2} s^{-2}-2 \nu, & \text { if } t \leq \tau s^{2}|1-s|^{-1}, \\
2 \tau|1-s|+\nu t-2 \nu, & \text { if } t>\tau s^{2}|1-s|^{-1} .\end{cases}
\end{aligned}
$$

$H(s, t)$ is continuous in $(s, t) \in \mathbb{R} \times(0,2)$, and there exists some $\left(s_{1}\right.$, $\left.t_{1}\right) \in \mathbb{R} \times[0,2]$ such that $t_{1}=\tau s_{1}^{2}\left|1-s_{1}\right|^{-1}$ and $H\left(s_{1}, t_{1}\right)=\inf \{H(s, t) \mid$ $\left.t>\tau s^{2}|1-s|^{-1}, \quad 0<t<2, \quad s \in \mathbb{R}\right\}$. Noting $\tau^{2} s^{2} t^{-1}+t(1-s)^{2} s^{-2} \geq 2 \tau|1-s|$ for $(s, t) \in \mathbb{R} \times(0,2)$, we have

$$
\begin{aligned}
& \inf \{H(s, t) \mid s \in \mathbb{R}, 0<t<2\} \\
& =\inf \left\{\tau^{2} s^{2} t^{-1}+\nu t+t(1-s)^{2} s^{-2}-2 \nu \mid s \in \mathbb{R}, 0<t<2\right\} .
\end{aligned}
$$

Here we have

$$
\begin{aligned}
& \tau^{2} s^{2} t^{-1}+\nu t+t(1-s)^{2} s^{-2}-2 \nu \\
& =t^{-1} s^{-2}\left\{\nu s^{2}+(1-s)^{2}\right\}\left[t-\nu s^{2}\left\{\nu s^{2}+(1-s)^{2}\right\}^{-1}\right]^{2} \\
& \quad+t^{-1} s^{2} \tau^{2}(\nu+1)\left\{\nu s^{2}+(1-s)^{2}\right\}^{-1}\left\{s-(\nu+1)^{-1}\right\}^{2} \\
& \quad+t^{-1} s^{2}\left\{\nu s^{2}+(1-s)^{2}\right\}^{-1} \nu(\nu+1)^{-1}\left\{\tau^{2}-\nu(\nu+1)\right\} .
\end{aligned}
$$

Then $\inf \{H(s, t) \mid s \in \mathbb{R}, 0<t<2\}<0$ is equivalent to $\tau^{2}<\nu(\nu+1)$, which leads to $\lambda>2^{-1} a\left[\left\{a^{2}+4 b^{2}\right\}^{1 / 2}-a\right]$.

\section{References}

[1] Agmon, S., Lower bounds for solutions of Schrödinger-type equations in unbounded domains, Proceedings of the International Conference of Functional Analysis and Related Topics, Tokyo, (1969), 216-224.

[2] Lower bounds for solutions of Schrödinger equations, J. Analyse Math. 23 (1970), 1-25.

[3] Eastham, M. S.P. and Kalf, H., Schrödinger-type operators with continuous spectra, Research notes in mathematics 65, Pitman Advanced Publishing Program, 1982.

[4] Ikebe, T. and Uchiyama, J., On the asymptotic behavior of eigenfunctions of secondorder elliptic operators, J. Math. Kyoto Univ. 11 (1971), 425-448.

[5] Kato, T., Growth properties of solutions of the reduced wave equation with a variable coefficient, Comm. Pure Appl. Math. 12 (1959), 403-425.

[6] Mochizuki, K., Growth properties of solutions of second-order elliptic differential equations, J. Math. Kyoto Univ. 16 (1976), 351-373.

[7] Uchiyama, J., Lower bounds of growth order of solutions of Schrödinger equations with homogeneous potentials, Publ. RIMS, Kyoto Univ. 10 (1975), 425-444.

[8] Decay order of eigenfunctions of second-order elliptic operators in an 
unbounded domain, and its applications, Publ. RIMS, Kyoto Univ. 22 (1986), 10791104.

[9] Uchiyama, J., Decay order of eigenfunctions of second-order elliptic operators in an unbounded domain, and its applications, Proceedings of Conference on Spectral and Scattering theory for differential operators, edited by S. Ito et al. (1986), 206-213.

[10] - Examples of exponential decay of eigenfunctions of magnetic Schrödinger operators, to appear on Proceedings of UAB / UA International Conference on Differential equations and mathematical Physics, edited by I. Knowles and Y. Saito.

[11] Lower bounds of decay order of eigenfunctions of second-order elliptic operators, Publ. RIMS, Kyoto Univ. 21 (1985), 1281-1297.

[12] Yamada, O., A note on the non-existence of eigenvalues of Schrödinger equations, Memoirs of the Research Institute of Science and Engineering, Ritsumeikan Univ., 32 (1977), $1-6$. 